\title{
Deep Learning in Cancer Diagnosis and Prognosis Prediction: A Minireview on Challenges, Recent Trends, and Future Directions
}

\author{
Ahsan Bin Tufail, ${ }^{1,2}$ Yong-Kui Ma ${ }^{D},{ }^{1}$ Mohammed K. A. Kaabar $\mathbb{D}^{3,4}{ }^{3}$ Francisco Martínez, ${ }^{5}$ \\ A. R. Junejo, ${ }^{6}$ Inam Ullah $\mathbb{D}^{7},{ }^{7}$ and Rahim Khan $\mathbb{D}^{1}$ \\ ${ }^{1}$ School of Electronics and Information Engineering, Harbin Institute of Technology, Harbin 150001, China \\ ${ }^{2}$ Department of Electrical and Computer Engineering, COMSATS University Islamabad, Sahiwal Campus, Sahiwal 57000, Pakistan \\ ${ }^{3}$ Gofa Camp, Near Gofa Industrial College and German Adebabay, Nifas Silk-Lafto, 26649 Addis Ababa, Ethiopia \\ ${ }^{4}$ Institute of Mathematical Sciences, Faculty of Science, University of Malaya, Kuala Lumpur 50603, Malaysia \\ ${ }^{5}$ Department of Applied Mathematics and Statistics, Technological University of Cartagena, Cartagena 30203, Spain \\ ${ }^{6}$ School of Control Science and Control Engineering, Harbin Institute of Technology, Harbin 150001, China \\ ${ }^{7}$ College of Internet of Things (IoT) Engineering, Hohai University (HHU), Changzhou Campus, 213022, China
}

Correspondence should be addressed to Yong-Kui Ma; yk_ma@hit.edu.cn,

Mohammed K. A. Kaabar; mohammed.kaabar@wsu.edu, and Inam Ullah; inam.fragrance@gmail.com

Received 20 August 2021; Revised 30 September 2021; Accepted 5 October 2021; Published 31 October 2021

Academic Editor: Iman Yi Liao

Copyright (c) 2021 Ahsan Bin Tufail et al. This is an open access article distributed under the Creative Commons Attribution License, which permits unrestricted use, distribution, and reproduction in any medium, provided the original work is properly cited.

\begin{abstract}
Deep learning (DL) is a branch of machine learning and artificial intelligence that has been applied to many areas in different domains such as health care and drug design. Cancer prognosis estimates the ultimate fate of a cancer subject and provides survival estimation of the subjects. An accurate and timely diagnostic and prognostic decision will greatly benefit cancer subjects. DL has emerged as a technology of choice due to the availability of high computational resources. The main components in a standard computer-aided design (CAD) system are preprocessing, feature recognition, extraction and selection, categorization, and performance assessment. Reduction of costs associated with sequencing systems offers a myriad of opportunities for building precise models for cancer diagnosis and prognosis prediction. In this survey, we provided a summary of current works where DL has helped to determine the best models for the cancer diagnosis and prognosis prediction tasks. DL is a generic model requiring minimal data manipulations and achieves better results while working with enormous volumes of data. Aims are to scrutinize the influence of DL systems using histopathology images, present a summary of state-of-the-art DL methods, and give directions to future researchers to refine the existing methods.
\end{abstract}

\section{Introduction}

Cancer is defined as abnormal cell growth that arises from any body organ. In essence, further growth of the cells in these organs is saturated. These silent and saturated cells are increased at a rapid rate till either their removal through a physical procedure such as surgery, medication, use of hormonal therapy, or radiation therapy or their disappearance on their own naturally. The natural disappearance of cancer cells can happen in cancers related to kidney or melanomas. These cells can be screened using tools such as colonoscopy or pap smear examination or using mammograms. There are more than 150 different kinds of cancer, and there is a lack of strategies to cure them in their early stages. Cancer stem cells are an effective way to form stromal cells thus paving a way for the cure of cancers. Apart from stem cells, WNT16B protein also increases resistance against cancer along with chemotherapy. Therapies such as laser therapy and cryotherapy are some of the most vibrant approaches to treat cancer. Some of the most prevalent types of cancers worldwide include lip, oral cavity, breast and cervical, and thyroid cancers. On the other hand, rare cancers such as osteosarcoma, Ewing's sarcoma, male breast cancer, gastrointestinal stromal tumors, chondrosarcoma, mesothelioma, 
adrenocortical carcinoma, cholangiocarcinoma, kidney chromophobe carcinoma, pheochromocytoma and paraganglioma, sarcoma, and ependymoma made up more than $20 \%$ of cancer cases and are rare types of cancers [1-4].

Cancer is a disease of genes. The process of replication, mitosis, and bombardment by oxygen cells bring continuous changes in normal and cancer cells. This process begins at the birth of a cancer cell and goes on till death. During this process, cancer cell gains mass using stromal support cells, immune cells, and endothelial cells. These cells become a part of cancer mass due to factors like stress ligands and antigens. Other emblems of cancer-based cellular stress are proteotoxicity, metabolic changes, and displaced acids of nucleotides. Another pattern of genes that drive them is chromosomes. They are drivers of a cell's nucleus. The human body has around 20,000 genes in somatic cells, and their study known as cytogenetics has seen large strides of progress over the past several decades where it is now possible to build a 3D model of chromosomes [5-7].

Sugar is an important constituent of tumor cells that fuels the rapid growth of these cells. They are an important part of the diet of cancer cells, and their growth ensures the formation of new clones. Bacteria and microbial cells colonize the human body. Microbial cells are estimated to be as abundant as human cells, yet their genome is roughly 100 times the human genome, providing significantly more genetic diversity. Helicobacter pylori, Chlamydia trachomatis, Salmonella enterica serovar typhi, Fusobacterium nucleatum, enterotoxigenic Bacteroides fragilis, Koribacteraceae, etc., are some of the most prominent bacteria that are associated with cancer. Apoptosis and necroptosis are two avenues of programmed cell death [8-10].

Cancer has long inspired fears. In the distant past, physicians related depression or melancholic humour to cancer's pathogenesis. It was believed that melancholy could give rise to a tumor as people attributed their cancer to sadness. Recently, inflammation and nonspecific immune activation are found to be key factors in the pathophysiology of depression related to cancer. Urban centers are at an increasing risk of cancer-related risks due to factors like nutrition; infections such as sea turtle fibropapillomatosis and feline immunodeficiency virus; urban chemical pollution such as carcinogens, polychlorinated biphenyls, glutathione, and urethane-induced adenomas; light and noise pollution such as suppression of pineal melatonin production; changes in survival; and life history strategies [11-13].

Deep learning (DL) has seen phenomenal growth in recent years in the use of artificial intelligence allowing complex computational models to represent abstractions gathered from data with wide applications in speech processing, visual processing, and other domains. These methods work by discovering fine structures in large and often complex datasets using a backpropagation algorithm. Compared to DL, conventional methods such as machine learning-based methods have limitations in processing natural data in its basic form without preprocessing [14].

Convolutional Neural Networks (CNNs) are DL systems equipped with the power to learn invariant features. CNNs have filter banks, feature pooling layers, batch normalization layers, dropout layers and dense layers that work in harmony to create patterns for different object recognition tasks such as detection, segmentation, and classification. CNNs have multilevel hierarchies where the distribution of inputs changes during the process of training. Preprocessed inputs, such as those obtained through the process of whitening, etc., are highly desirable to obtain better performances across tasks [15]. CNNs have many different variants such as those offering shorter connections, for example, DenseNet architecture, which offer advantages in terms of feature circulation and offer substantial reduction in hyperparameters to build efficient architectures [16]. The focal and nonfocal electroencephalogram signals in tunable $Q$-factor wavelet transform domain have been investigated and identified with the help of feature selection and neural network methods [17]. A recent study concerning the low-density parity-check (LDPC) codes for Internet of things networks has been conducted via a novel technique for obtaining the first two minima of check-node update operation of the min-sum-LDPC decoder [18]. In addition, a review of future robust networks including various scenario for $6 \mathrm{G}$ has been discussed in [19].

Other types of CNN architectures that have gained popularity in recent years are ResNets, Xception, and GoogLeNet architectures. The need for these networks arises due to degradation in performances across tasks when the network is getting deeper, the need for multiscale processing, and the search for better architectures with less number of parameters [20-23].

Another issue that holds considerable importance in DL is the ability of an architecture to store information over extended time intervals. One solution proposed for this problem is Long Short-Term Memory (LSTM). LSTM architecture works by enforcing consistent error flow that is nonglobal in space and time through states of specialized units [24].

Another idea worth mentioning in DL is the notion of transfer learning. In transfer learning, features extracted from deep CNNs are repurposed to new and novel tasks. The need arises because generic tasks may differ by a wide margin from the original tasks due to which there may be insufficient labelled or other data to train or adapt a DL architecture to new tasks. Using transfer learning, features can be adapted to have sufficient generalization expression using simple techniques reliably [25-27].

Finding better architecture design parameters for DL models is a problem worth considering. Reinforcement learning methods can help in this task. An inspirational example is NASNet architecture that uses a number of different network topologies to find repeated motifs that can be combined in series to handle inputs of varying spatial dimensions and depth [28, 29].

This paper presents an overview of DL methods for the task of cancer diagnosis, prognosis, and prediction. The aim is to highlight the differences between different model constructions and to provide limitations and future perspectives for further exploration of this exciting domain.

The rest of the paper is organized as follows. Section 2 presents the gist behind the selection of studies that are 
made a part of this survey article. Section 3 presents an overview of publically available datasets for cancer research followed by the description of current applications of DL in cancer diagnosis, prognosis, and prediction tasks in Section 4. Section 5 presents the discussion covering limitations of the existing methods, perspectives, and some directions for future work. Finally, Section 6 concludes this work and proposes avenues for further research in this domain.

\section{Methodology}

The criterion used for the selection of articles for this minireview was language and authenticity of electronic sources. Articles written only in English language are made a part of this survey due to wide recognition of English as the language of scientific and biomedical domains. Years of sources of articles considered for this study range between 1997 and 2020. We used PubMed, Web of Science, IEEE Xplore, and Science Direct platforms to conduct the research. The search terms used were diagnosis of cancer, prognosis of cancer, prediction of cancer using DL, and transfer learning models.

\section{Publically Available Datasets for Cancer Research Using DL Methods}

In this section, we will provide a brief description of publically available datasets for cancer studies. We will briefly describe The Cancer Genome Atlas (TCGA) database, Rotterdam tumor bank, Study to Understand Prognoses and Preferences for Outcomes and Risks of Treatment (SUPPORT), Molecular Taxonomy of Breast Cancer International Consortium (METABRIC), MITOS-ATYPIA-14 dataset, Tumor Proliferation Assessment Challenge (TUPAC) 2016 dataset, INbreast database, Lung Image Database Consortium and Image Database Resource Initiative (LIDC-IDRI) datasets, Lung Nodule Analysis (LUNA16) dataset, Breast Cancer Histopathological Image Classification (BreakHis) dataset, 2015 Bioimaging breast histology classification challenge, Cancer Metastases in Lymph Nodes Challenge breast cancer metastasis detection (CAMELYON) dataset, PatchCamelyon dataset, 2018 International Conference on Image Analysis and Recognition (ICIAR) dataset, MITOS12 dataset, Leukemia microarray gene data, Gene Expression Omnibus repository, BioGPS data portal, The Cancer Imaging Archive (TCIA), Genomic Data Commons (GDC), Therapeutically Applicable Research to Generate Effective Treatments (TARGET), 1000 Genomes Project, Kvasir dataset, University of California Santa Barbara Bio Segmentation Benchmark (UCSB-BB) dataset, and the Multimodal Brain Tumor Image Segmentation Benchmark (BRATS) dataset.

(1) TCGA Database. Beginning in 2006, TCGA is a result of a joint partnership between the National Cancer Institute and the National Human Genome Research Institute characterizing over 20,000 primary cancer and matched normal samples spanning 33 cancer types such as acute myeloid leukemia, adrenocortical carcinoma, breast lobular carcinoma, and uveal melanoma. The total number of cases in this database is approximately 11,125 . It also contains cases of rare types of cancers. This database is available at https://www.cancer.gov/aboutnci/organization/ccg/research/structural-genomics/ tcga (accessed on September 24, 2021).

(2) Rotterdam Tumor Bank. This dataset [30] is comprised of 2982 primary breast cancer patients of whom 1546 are positive cases, i.e., they had the disease. Different factors such as estrogen receptors, progesterone receptors, hormonal treatment, number of positive lymph nodes, differentiation grade, and tumor size characterize this dataset. An R package of this dataset can be accessed at https://stat .ethz.ch/R-manual/R-devel/library/survival/html/ rotterdam.html (accessed on September 24, 2021).

(3) SUPPORT Database. This database [31], gathered with the support of five teaching hospitals in the United States, is comprised of 9105 adults with an overall 6 -month mortality rate of $47 \%$. Subjects are recruited in two phases. Phase I recruited 4301 patients while phase II recruited 4804 patients. In phase II, the intervention group has 2652 subjects while the control group has 2152 subjects. Patients are diagnosed with one of nine life-threatening diagnoses.

(4) METABRIC Dataset. This dataset [32] is comprised of 2509 primary breast tumor subjects with 548 matched normal subjects. There are 2506 breast cancer subjects and 3 breast sarcoma subjects. Subjects with breast invasive ductal carcinoma are the most frequently occurring in this dataset while subjects of metaplastic breast cancer and breast angiosarcoma are the least frequently occurring classes. In total, there are eight classes of cancer subjects. This dataset is available at https://www.cbioportal .org/study/summary?id=brca_metabric (accessed on September 24, 2021).

(5) MITOS-ATYPIA-14 Dataset. This dataset contains histological images of breast cancer for the detection of mitotic cells and for the evaluation of nuclear atypia score for the prognosis of breast cancer. These annotations are provided by two senior and three junior pathologists. The dataset provides samples of haematoxylin and eosin-stained slides with the size of $1539 \times 1376$ pixels at $20 x$ and $40 x$ magnification levels. For every slide, the pathologists selected several frames at $\times 10$ magnification which are further subdivided into four frames at $\times 20$ magnification which are further subdivided into four frames at $\times 40$ magnification. Evaluation metrics for mitosis are the number of detected mitosis, number of true positives, number of false positives, number of false negatives, sensitivity, precision, and $F_{1}$-value. This dataset is available at https://mitos-atypia-14.grand-challenge.org/Home/ (accessed on September 24, 2021).

(6) TUPAC 2016 Dataset. This dataset [33] provides a way to predict tumor proliferation scores from 
whole-slide images. The challenge dataset is made up of 500 training and 321 testing breast cancer histology whole-brain slides. This dataset is designed to fulfill three purposes. The first one is to predict mitotic scores while the second one is to predict gene expression-based proliferation scores. A third task was later added to the challenge for mitosis detection.

(7) INbreast Dataset. This breast cancer dataset [34] has a total of 115 cases and is made up of fullfield digital mammograms. The number of images of these cases is 410 . In these 115 cases, 90 cases are from women with both breasts affected while 25 cases represent mastectomy patients. Several types of lesions such as masses, calcifications, asymmetries, multiple findings, normal, and distortions are included. Eight cases also have images acquired at different timings.

(8) LIDC-IDRI Database. Initiated by the National Cancer Institute (NCI), this dataset [35] of Computed Tomography (CT) scans contains 1018 cases of three categories: nodule $\geq 3 \mathrm{~mm}$, nodule $<3 \mathrm{~mm}$, and nonnodule $\geq 3 \mathrm{~mm}$. A two-phase image annotation process was performed by four experienced thoracic radiologists. The goal of this dataset is to identify as completely as possible all lung nodules in each CT scan. This dataset is available at https:// wiki.cancerimagingarchive.net/display/Public/LIDCIDRI\# 1966254194132fe653e4a7db00715f6f775c012 (accessed on September 24, 2021).

(9) LUNA16 Dataset. Collected from the LIDC-IDRI dataset, the LUNA16 [36] dataset is designed for the detection of pulmonary nodules from CT scans. The scans where slices were thicker than $2.5 \mathrm{~mm}$ were excluded. It facilitates lung nodule segmentation by providing the option of multiple candidates per nodule. In total, this dataset includes 888 CT scans. Irrelevant findings that were not made a part of this dataset include nonnodules, nodules $<3 \mathrm{~mm}$, and nodules annotated by only 1 or 2 radiologists. This dataset is available at https://luna16.grand-challenge .org/Data/ (accessed on September 24, 2021).

(10) BreakHis Dataset. This dataset [37] of breast cancer subjects contains 9109 microscopic images. These images of tumor tissue are collected from 82 subjects at four different magnification levels which are $40 \mathrm{x}, 100 \mathrm{x}, 200 \mathrm{x}$, and $400 \mathrm{x}$. It contains 2480 benign and 5429 malignant samples. These samples are stored in PNG format. The resolution of each sample is $700 \times 460$ pixels, 3 -channel RGB with eight-bit depth in each channel. This database resulted from the collaboration of the P\&D Laboratory Pathological Anatomy and Cytopathology, Parana, Brazil, and Laboratory of Vision, Robotics, and Imaging, Federal University of Parana, Brazil. Benign tumors are slow-growing and remain localized to a region while malignant tumors can spread to distant regions and possess the ability to destroy adjacent structures which may cause death. This dataset is available at https://web.inf.ufpr.br/vri/ databases/breast-cancer-histopathological-databasebreakhis/ (accessed on September 24, 2021).

(11) 2015 Bioimaging Breast Histology Classification Challenge Dataset. This dataset [38] of breast cancer subjects has four classes which are normal, benign, in situ carcinoma, and invasive carcinoma. It has highresolution, uncompressed, and annotated H\&E stain slides. The images have a resolution of $2040 \times 1536$ pixels. It supports both image and patch-wise classification tasks. This dataset is available at https:// rdm.inesctec.pt/dataset/nis-2017-003 (accessed on September 24, 2021).

(12) CAMELYON Dataset. This dataset [39] is designed for breast cancer metastasis detection and classification in whole-slide images of histological lymph nodes. It facilitates patient-level analysis by combining the assessment of several lymph node slides into one outcome for direct deployment in a clinical setting which will facilitate pathologists while at the same time reducing the subjectivity in diagnosis. It contains 1399 unique whole-slide images of lymph nodes which have a slide-level label indicating whether it contains no metastases, macrometastases, micrometastases, or isolated tumor cells. In addition, the dataset has detailed contours drawn by experts for all metastases in 209 whole-slide images. This dataset is available at https://camelyon17.grandchallenge.org (accessed on September 24, 2021).

(13) PatchCamelyon Dataset. This dataset [40] contains histopathologic scans of breast cancer lymph node sections. Each image in this dataset is annotated with a label to indicate the presence of metastatic tissue. It contains 327,680 color images with a resolution of $96 \times 96$ pixels. It is trainable on a single GPU. For size comparisons, it is bigger than CIFAR10 and smaller than the ImageNet dataset. This dataset is available at https://www.tensorflow .org/datasets/catalog/patch_camelyon (accessed on September 24, 2021).

(14) 2018 ICIAR Dataset. This dataset is composed of haematoxylin and eosin-stained breast histology microscopy and whole-slide images. The images are labelled as normal, benign, in situ carcinoma, or invasive carcinoma. There are a total of 400 microscopy images with 100 images per class stored with .tiff extension. The microscopy images are color images with a dimension of $2048 \times 1536$ pixels. The whole-slide images are color images with a dimension of $42113 \times 62625$ pixels and are stored in .svs format with pixel-wise labels. This dataset is available at https://iciar2018-challenge.grand-challenge.org/ Dataset/ (accessed on September 24, 2021). 
(15) MITOS12 Dataset. This dataset [41] contains 50 breast cancer biopsy slides at a 40x magnification level with more than 300 mitoses in these slides. The dimensions of these images are $2084 \times 2084$ pixels and $2252 \times 2250$ pixels. This dataset is helpful with mitotic count to estimate the aggressiveness of the breast tumor. This dataset is available at http:// ludo17.free.fr/mitos_2012/dataset.html (accessed on September 24, 2021).

(16) Leukemia Microarray Gene Data. This dataset [42] contains gene expression data from 60 bone marrow samples of patients belonging to acute lymphoblastic leukemia, acute myeloid leukemia, chronic lymphocytic leukemia, chronic myeloid leukemia, and healthy bone marrow. Microarray technology has been instrumental in genome-wide expression studies especially as the knowledge of metazoan genomes is improving. Further information about this dataset is available at https://www.bioconductor.org/packages/ devel/data/experiment/manuals/leukemiasEset/man/ leukemiasEset.pdf (accessed on September 24, 2021).

(17) Gene Expression Omnibus Repository. This repository [43] provides comprehensive sets of microarray, next-generation sequencing, and other genomic data to facilitate research in different types of cancers. Further information about this repository is available at https://www.ncbi.nlm.nih.gov/geo/ (accessed on September 24, 2021).

(18) BioGPS Data Portal. This portal [44] supports eight species which are humans, mouse, rat, fruitfly, nematode, zebrafish, thale-cress, frog, and pig to facilitate research in genes. Many different kinds of cancers are supported such as lung cancer, breast cancer, esophageal cancer, thyroid cancer, pancreatic cancer, colorectal cancer, and colon cancer. This portal is available at http://biogps.org/\#goto= welcome (accessed on September 24, 2021).

(19) TCIA. This service [45] provides deidentification and hosting of a large archive of medical images of cancer for public access using different modalities such as Magnetic Resonance Imaging (MRI), $\mathrm{CT}$, and digital histopathology. It also provides supporting data such as patient outcomes, treatment details, and genomics. This service is available at https://www.cancerimagingarchive.net (accessed on September 24, 2021).

(20) GDC. This portal provides genomic, clinical, and biospecimen data for different types of cancers such as bone marrow, breast, eye, skin, lung, liver, and nervous system. It supports cancer research initiatives such as TCGA and TARGET. This portal is available at https://gdc.cancer.gov (accessed on September 24, 2021).

(21) TARGET. This program provides vast amounts of genomic data to estimate molecular alterations in childhood cancers. The goal is to use data for the development of effective, less toxic therapies. It drives research in acute lymphoblastic leukemia, acute myeloid leukemia, kidney tumors, neuroblastoma, osteosarcoma, etc. Further information is available at https://ocg.cancer.gov/programs/target\# (accessed on September 24, 2021).

(22) 1000 Genomes Project. The aim of this project [46] is to find common genetic variants by taking advantage of developments in sequencing technology. It helps in sequencing a large number of people to provide a comprehensive resource on human genetic variation. Cell lines and DNA are available for all 1000 Genomes samples. These samples are completely anonymous with no associated medical data. Further information about this project is available at https://www.internationalgenome.org/ 1000-genomes-summary (accessed on September 24, 2021).

(23) Kvasir Dataset. This dataset [47] is accessible at https://dl.acm.org/do/10.1145/3193289/abs/ (accessed on September 24, 2021). It is designed to facilitate research in gastrointestinal (GI) tract cancer. The initial dataset consists of 4000 annotated images belonging to 8 classes with 500 images per class. The anatomical landmarks are Z-line, pylorus, and cecum, while the pathological finding includes esophagitis, polyps, and ulcerative colitis. Resolution of images ranges from $720 \times 576$ up to $1920 \times 1072$ pixels. This dataset continues to play an important role in deep learning research.

(24) UCSB-BB Dataset. This dataset [48] contains images of human, monkey, and cat species at subcellular, cellular, and tissue levels with resolutions ranging from $300 \times 200$ to $1024 \times 1024$ pixels. There are 58 images of breast cancer belonging to malignant/benign classes in humans with sizes of $896 \times 768$ and $768 \times 512$ at the cellular level associated with ground truth data. This dataset is available at https://bioimage.ucsb.edu/ research/bio-segmentation (accessed on September 24, 2021).

(25) BRATS Dataset. This dataset [49] is composed of clinical and synthetic images. The clinical data has $65 \mathrm{MRI}$ scans of low- and high-grade glioma patients. Four MRI contrasts which are T1, T1c, T2, and FLAIR are represented by clinical data. The synthetic data has 35 high-grade and 30 low-grade glioma subjects. It is aimed at facilitating segmentation of tumors and patient survival through prediction and differentiation between tumor recurrence and progression. This dataset is available at https://www .med.upenn.edu/cbica/brats2020/ (accessed on September 24, 2021).

Table 1 displays a summary of the databases/service$\mathrm{s} /$ projects to facilitate cancer research covered in this section. 
TABLE 1: Summary of the datasets for cancer research.

\begin{tabular}{|c|c|c|c|}
\hline Dataset/service/project & Link & Type(s) of cancer(s) & Description \\
\hline TCGA database & $\begin{array}{l}\text { https://www.cancer.gov/aboutnci/organization/ccg/ } \\
\text { research/structural-genomics/tcga }\end{array}$ & Multiple & $\begin{array}{l}33 \text { cancer types, total no. of cases } \\
\text { is } 11125\end{array}$ \\
\hline Rotterdam tumor bank & $\begin{array}{c}\text { https://stat.ethz.ch/R-manual/R-devel/library/survival/ } \\
\text { html/rotterdam.html }\end{array}$ & Breast cancer & $\begin{array}{l}2982 \text { primary breast cancer } \\
\text { patients; } 1546 \text { are positive cases }\end{array}$ \\
\hline SUPPORT database & [31] & Multiple & $\begin{array}{l}9105 \text { adults, an overall } 6 \text {-month } \\
\text { mortality rate of } 47 \%\end{array}$ \\
\hline METABRIC dataset & $\begin{array}{c}\text { https://www.cbioportal.org/study/summary?id=brca } \\
\text { metabric }\end{array}$ & Breast cancer & $\begin{array}{l}2509 \text { primary breast tumor } \\
\text { subjects, } 548 \text { matched normal } \\
\text { control subjects }\end{array}$ \\
\hline $\begin{array}{l}\text { MITOS-ATYPIA-14 } \\
\text { dataset }\end{array}$ & https://mitos-atypia-14.grand-challenge.org/Home/ & Breast cancer & $\begin{array}{c}\text { Resolution of } 1539 \times 1376 \text { pixels } \\
\text { at } 20 x \text { and } 40 x \text { magnification } \\
\text { levels }\end{array}$ \\
\hline TUPAC 2016 dataset & {$[33]$} & Breast cancer & $\begin{array}{l}500 \text { training and } 321 \text { testing } \\
\text { breast cancer histology whole- } \\
\text { brain slides }\end{array}$ \\
\hline INbreast dataset & {$[34]$} & Breast cancer & $\begin{array}{c}\text { Total of } 115 \text { cases and } 410 \\
\text { images }\end{array}$ \\
\hline LIDC-IDRI database & $\begin{array}{l}\text { https://wiki.cancerimagingarchive.net/display/Public/ } \\
\text { LIDCIDRI\#1966254194132fe653e4a7db00715f6f775c012 }\end{array}$ & Lung cancer & $\begin{array}{l}\text { CT scans of } 1018 \text { subjects, three } \\
\text { categories (i) nodule } \geq 3 \mathrm{~mm} \text {, (ii) } \\
\text { nodule }<3 \mathrm{~mm} \text {, and (iii) } \\
\text { nonnodule } \geq 3 \mathrm{~mm}\end{array}$ \\
\hline LUNA16 dataset & https://luna16.grandchallenge.org/Data/ & Lung cancer & $\begin{array}{l}888 \text { CT scans, facilitates } \\
\text { segmentation studies }\end{array}$ \\
\hline BreakHis dataset & $\begin{array}{l}\text { https://web.inf.ufpr.br/vri/databases/breast- } \\
\text { cancerhistopathological-database-breakhis/ }\end{array}$ & Breast cancer & $\begin{array}{l}9109 \text { microscopic images; four } \\
\text { different magnification levels } \\
\text { which are 40x, 100x, 200x, and } \\
400 x \text { collected from } 82 \text { subjects }\end{array}$ \\
\hline $\begin{array}{l}2015 \text { Bioimaging } \\
\text { Breast Histology } \\
\text { Classification } \\
\text { Challenge }\end{array}$ & https://rdm.inesctec.pt/dataset/nis-2017-003 & Breast cancer & $\begin{array}{l}\text { Four classes which are normal, } \\
\text { benign, in situ carcinoma, and } \\
\text { invasive carcinoma; resolution } \\
\text { of } 2040 \times 1536 \text { pixels }\end{array}$ \\
\hline CAMELYON dataset & https://camelyon17.grand-challenge.org & Breast cancer & $\begin{array}{l}\text { Facilitates patient-level analysis; } \\
1399 \text { unique whole-slide images; } \\
\text { no metastases, macrometastases, } \\
\text { micrometastases, and isolated } \\
\text { tumor cells }\end{array}$ \\
\hline PatchCamelyon dataset & $\begin{array}{c}\text { https://www.tensorflow.org/datasets/catalog/patch_ } \\
\text { camelyon }\end{array}$ & Breast cancer & $\begin{array}{l}327,680 \text { color images with } \\
\text { resolution of } 96 \times 96 \text { pixels; } \\
\text { bigger than CIFAR10 and } \\
\text { smaller than ImageNet dataset }\end{array}$ \\
\hline 2018 ICIAR dataset & https://iciar2018-challenge.grand-challenge.org/Dataset/ & Breast cancer & $\begin{array}{l}\text { Represent normal, benign, in } \\
\text { situ carcinoma, and invasive } \\
\text { carcinoma; } 400 \text { microscopy } \\
\text { images with } 100 \text { images per class }\end{array}$ \\
\hline MITOS12 dataset & http://ludo17.free.fr/mitos_2012/dataset.html & Breast cancer & $\begin{array}{l}50 \text { biopsy slides; } 40 \mathrm{x} \\
\text { magnification level; more than } \\
\quad 300 \text { mitoses }\end{array}$ \\
\hline $\begin{array}{l}\text { Leukemia microarray } \\
\text { gene data }\end{array}$ & $\begin{array}{c}\text { https://www.bioconductor.org/packages/devel/data/ } \\
\text { experiment/manuals/leukemiasEset/man/leukemiasEset } \\
\text {.pdf }\end{array}$ & $\begin{array}{l}\text { Bone marrow } \\
\text { cancer }\end{array}$ & $\begin{array}{l}60 \text { bone marrow samples; acute } \\
\text { lymphoblastic leukemia, acute } \\
\text { myeloid leukemia, chronic } \\
\text { lymphocytic leukemia, chronic } \\
\text { myeloid leukemia, and healthy } \\
\text { bone marrow }\end{array}$ \\
\hline
\end{tabular}


TABLE 1: Continued.

\begin{tabular}{|c|c|c|c|}
\hline Dataset/service/project & Link & Type(s) of cancer(s) & Description \\
\hline $\begin{array}{l}\text { Gene Expression } \\
\text { Omnibus repository }\end{array}$ & https://www.ncbi.nlm.nih.gov/geo/ & Multiple & $\begin{array}{c}\text { Provides comprehensive sets of } \\
\text { microarray, next-generation } \\
\text { sequencing, and other genomic } \\
\text { data }\end{array}$ \\
\hline BioGPS data portal & http://biogps.org/\#goto=welcome & Multiple & $\begin{array}{l}\text { Supports eight species including } \\
\text { humans; supports different types } \\
\text { of cancers }\end{array}$ \\
\hline TCIA & https://www.cancerimagingarchive.net & Multiple & $\begin{array}{l}\text { Supports a large number of } \\
\text { modalities; supports data such as } \\
\text { patient outcomes, treatment } \\
\text { details, and genomics }\end{array}$ \\
\hline GDC & https://gdc.cancer.gov & Multiple & $\begin{array}{l}\text { Provides genomic, clinical, and } \\
\text { biospecimen data }\end{array}$ \\
\hline TARGET & https://ocg.cancer.gov/programs/target\# & Multiple & $\begin{array}{l}\text { Childhood cancers are } \\
\text { supported; provides vast } \\
\text { amounts of genomic data to } \\
\text { estimate molecular alterations }\end{array}$ \\
\hline 1000 Genomes Project & $\begin{array}{l}\text { https://www.internationalgenome.org/1000-genomes- } \\
\text { summary }\end{array}$ & Multiple & $\begin{array}{l}\text { Provides a comprehensive } \\
\text { resource on human genetic } \\
\text { variation }\end{array}$ \\
\hline Kvasir dataset & https://dl.acm.org/do/10.1145/3193289/abs/ & $\begin{array}{l}\text { Gastrointestinal } \\
\text { tract cancer }\end{array}$ & $\begin{array}{l}4000 \text { annotated images } \\
\text { belonging to } 8 \text { classes }\end{array}$ \\
\hline UCSB-BB dataset & https://bioimage.ucsb.edu/research/bio-segmentation & $\begin{array}{l}\text { Supports breast } \\
\text { cancer research in } \\
\text { human species }\end{array}$ & $\begin{array}{c}\text { Contains images of human, } \\
\text { monkey, and cat species at } \\
\text { subcellular, cellular, and tissue } \\
\text { levels }\end{array}$ \\
\hline BRATS dataset & https://www.med.upenn.edu/cbica/brats2020/ & Brain tumor & $\begin{array}{l}\text { MRI scans of } 65 \text { subjects each in } \\
\text { clinical and synthetic datasets, } \\
\text { for brain tumor segmentation } \\
\text { task }\end{array}$ \\
\hline
\end{tabular}

\section{Current Applications of Deep Learning in Cancer Diagnosis, Prognosis, and Prediction}

In this section, we will discuss some current research trends in the domain of DL for cancer diagnosis, prognosis, and prediction tasks. We will cover techniques for the prognosis/prediction of tumors, breast cancer, and other types of cancer. In addition, we will also cover techniques for the segmentation/detection of breast cancer and other types of cancer. Furthermore, we will cover different methods for the classification of breast cancer and other types of cancer. We will also cover techniques for the classification, segmentation, and detection of brain tumors. Figure 1 shows histopathological views of some of the cancer subtypes that will be covered in this review article.

4.1. Prognosis/Prediction of Tumors, Breast Cancer, Skin Cancer, Head and Neck Cancer, Brain Cancer, Liver Cancer, Colorectal Cancer, Ovarian Cancer, and Other Types of Cancer. Petalidis et al. [50] reported a gene expression dataset for astrocytic tumors. They employed an Artificial Neural Network (ANN) algorithm to combine signatures from histopathological subclasses of these tumors in order to address the need for proper grading of these tumors. In this study, they found 59 genes which belong to three classes, namely, angiogenesis, lower-grade astrocytic tumor discrimination, and cell differentiation. They further report that these tumor subtypes have very high prognostic value, and they are missing in other studies reported in the literature. Finally, they report 11 classifiers that used genes to differentiate among primary/secondary subtypes of glioblastomas. They used a custom as well as independent dataset reporting accuracy of $96.15 \%$ to identify correct classes for these subtypes. Chi et al. [51] use morphometric features to compare prediction outcomes on two different breast cancer datasets. They report successful predictions with good and bad prognostic values. Here, good means that prognosis stands valuable even after five years while bad suggests less than five years. The authors in [52] conducted experiments in female breast carcinoma patients using a DL approach. They did prediction using a Cox regression model and gene expression datasets. They called their approach Survival Analysis Learning with MultiOmics Neural Networks (SALMON). They report that performance of SALMON is improved when more data is used to combine and simplify cancer biomarkers and gene expressions to enable prognosis prediction. Shimizu and Nakayama [53] conducted experiments to identify breast cancer genes for prognosis prediction using The Cancer Genome Atlas (TCGA) database. They identified 184 genes using artificial intelligence (AI), 


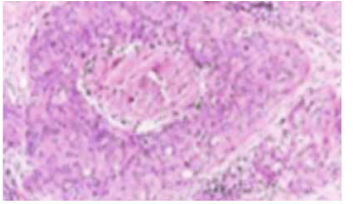

(a)

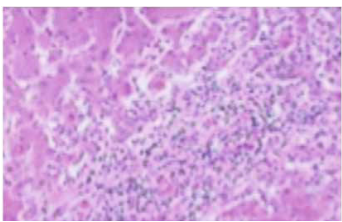

(e)

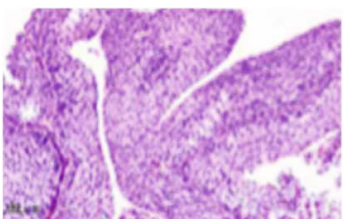

(i)

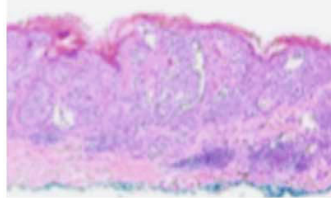

(b)

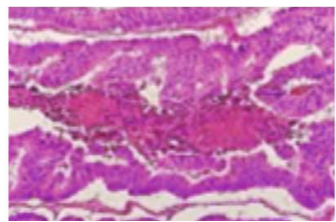

(f)

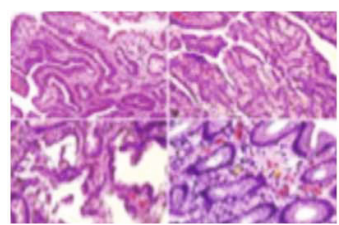

(j)

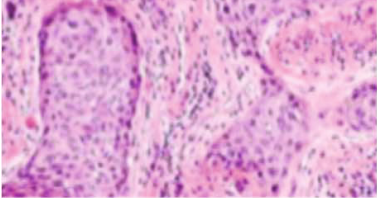

(c)

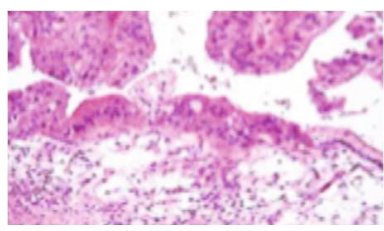

(g)

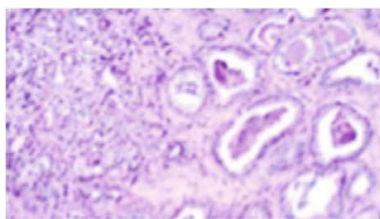

(k)

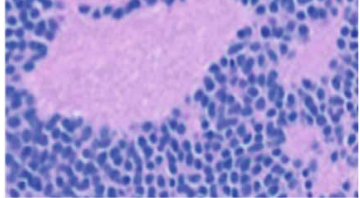

(d)

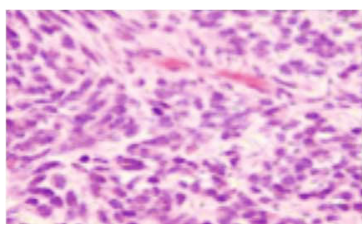

(h)

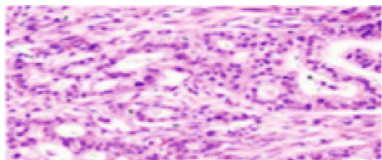

(1)

FIGURE 1: Histopathological images of different cancer subtypes: (a) breast cancer, (b) skin cancer, (c) head and neck cancer, (d) brain cancer, (e) liver cancer, (f) colorectal cancer, (g) ovarian cancer, (h) lung cancer, (i) bladder cancer, (j) gastric cancer, (k) prostate cancer, and (l) pancreatic cancer.

and for that purpose, they used random forest and neural network models. Furthermore, they used a molecular score for prognosis that uses only 23 of these genes. They confirmed that they have found potential drug targets in these genetic discoveries. The authors in [54] performed their experiments using malignant melanoma. They used a dataset with 1160 females and 786 males. They used an ANN architecture employing a flexible nonlinear structure for prognosis prediction of survival probabilities. They found the performance of their model to be at par with the Cox model with the advantage that it offers a flexible approach when analyzing data using a specified distributional form. Jing et al. [55] introduced a loss function combining a pairwise ranking loss and a mean squared error loss to optimize a DL model validated on four publically available datasets, such as the Worcester Heart Attack Study (WHAS), Rotterdam tumor bank, Study to Understand Prognoses and Preferences for Outcomes and Risks of Treatment (SUPPORT), and Molecular Taxonomy of Breast Cancer International Consortium (METABRIC). Their model achieved superior performance results than medical experts for nasopharyngeal carcinoma prognosis. Hao et al. [56] proposed a DL network predicting prognoses and describing complex biological pathways thus providing their model the power to interpret its outcomes. They conducted experiments for prediction in glioblastoma multiforme brain cancer from TCGA database. Their model achieves an Area under the Curve (AUC) of $0.6622 \pm 0.013$ and an $F_{1}$ score of $0.3978 \pm 0.016$ outperforming other models such as Logistic Least Absolute Shrinkage and Selection Operator (LASSO), Random LASSO, Support Vector Machines (SVMs), and a dropout neural network model which shows the superiority of their approach. The authors [57] put forward a multimodal DL network integrating multidimensional data. Their model combined gene expression data, alteration data, and other forms of clinical data achieving better performance than models with 1D data and other approaches. Chaudhary et al. [58] proposed the DL-based approach based on a combination of RNA and methylation data from TCGA to model hepatocellular carcinoma subjects. Their model achieves a concordance index of 0.68 and a $p$ value of $7.13 \times 10^{-6}$. They found that TP53 mutations, KRT19 and EPCAM stemness markers, and Wnt and Akt signaling pathways are associated with more aggressive subtypes. The authors in [59] proposed a DL model combining CNN and Recurrent Neural Network (RNN) architectures for the prediction of colorectal cancer subjects using digitized haematoxylin-eosin-stained tumor tissue microarray samples. In the low- and high-risk patients, their model achieved a hazard ratio of 2.3 for visual assessment of histological tissues and a hazard ratio of 1.65 on the whole-slide level for both low- and high-risk subjects. Wang et al. [60] come up with a DL model to predict serous ovarian cancer subjects by extracting prognostic biomarkers from CT images. They further proposed a combined DL and Cox hazards model and achieved a concordance index of 0.713 and 0.694 for individual and three years of recurrence probability of subjects, respectively. The authors in [61] used a DL-based ANN model from transcriptomics data. They deployed TCGA datasets of RNA sequences belonging to ten different kinds of cancers. Their model achieved superior or equal level performance at both the pathway and gene levels. The authors in [62] came up with a DL model combining a Cox proportional hazards model with one of the best performing survival methods. They conducted experiments on WHAS, METABRIC, and SUPPORT datasets achieving good prediction performance levels for personalized treatment recommendations. Mobadersany et al. [63] predicted time-to-event results from histopathology images and gene- 
based biomarkers using CNNs as DL models from glioma and glioblastoma cohorts of TCGA. They used a samplingand filtering-based approach for the improvement of their predictions by not taking into account the intratumoral heterogeneity. Their model achieved a median concordance index of 0.754 surpassing other state-of-the-art approaches. The authors in [64] developed a DL-based approach using CNNs to predict the survival of mesothelioma cancer subjects. They used TCGA and a French source to test their approach. They achieved a concordance index of 0.656 on the TCGA cohort surpassing the performance of human experts and found key regions in the stroma that are associated with inflammation and cellular diversity. Liu et al. [65] modeled diagnostic prediction using DL models. The authors conducted their study on 27 diverse cancer types obtained from TCGA and Gene Expression Omnibus dataset. They successfully decoded $12 \mathrm{CpG}$ and 13 promoter markers. The CpG markers that they identified achieved a sensitivity of $100 \%$ in the prediction of prostate cancer samples while promoter markers achieved 92\% using cell-free deoxyribonucleic acid (DNA) methylation data.

Table 2 displays a summary of the studies for the task of prognosis and prediction of cancers covered in this subsection.

4.2. Segmentation/Detection of Breast Cancer, Lung Cancer, Bladder Cancer, and Other Types of Cancer. Yap et al. [66] used DL approaches for breast lesion detection using ultrasound images. They investigated the performance of LeNet, U-Net, and a pretrained AlexNet. They conducted their experiments on two custom datasets of 306 and 163 images termed dataset $\mathrm{A}$ and dataset $\mathrm{B}$, respectively. Their pretrained AlexNet-based model achieved the best overall performance by achieving an F-measure of 0.91 and 0.89 on both datasets. The authors in [67] come up with different variants of fully convolutional networks (FCNs) for the segmentation of lesions of breast cancer subjects. They tried an AlexNet-based FCN, as well as 8-, 16-, and 32-layered FCN models. To overcome the problem of data deficiency, they used transfer learning and pretraining on the ImageNet dataset. Their dataset has two classes, benign and malignant. They reported an average dice score of 0.7626 using FCN with 16 layers on benign lesions. Their model correctly recognized $89.6 \%$ of benign lesions and $60.6 \%$ of the malignant lesions successfully. Liu et al. [68] used DL to detect breast cancer in lymph node biopsies. They used 399 slides from the Camelyon 16 challenge dataset to achieve an AUC of 99\% at the slide level. They used a second custom dataset that has 108 slides to achieve an AUC of 99.6\%. As a preprocessing step, they used a color normalization procedure. The authors in [69] used different DL methods such as faster region CNN, ResNET-50, and DenseNet-201 architectures for breast cancer detection using histopathology images. They used three datasets to conduct their experiments which are International Conference on Pattern Recognition 2012, MITOS-ATYPIA-14, and Tumor Proliferation Assessment Challenge 2016 dataset. They achieved a precision of 0.876 on the International Conference on Pattern Recognition 2012 dataset, 0.848 on MITOS-ATYPIA-14, and a precision of 0.641 on the Tumor Proliferation Assessment Challenge
2016 dataset. As data augmentation methods, they employed horizontal and vertical flipping, translation, and resizing operations to artificially increase the size of datasets. Anuranjeeta et al. [70] used shape and morphological features derived from segmented images to detect cancer cells using a number of DL and machine learning-based models. They used J-Rip, logistic modal tree, rotation forest, multilayer perceptron, and other models trained by histopathological images. Rotation forest performed the best in cancerous/noncancerous detection achieving an accuracy of $85.7 \%$. The authors in [71] used a modified regional CNN method to efficiently determine mitosis in breast cancer using histopathological images. They employed subjects belonging to the 2014 International Conference on Pattern Recognition (ICPR) and TUPAC 2016 datasets in their study. They achieved 0.76 in precision on the TUPAC 2016 dataset. Zhou et al. [72] used a 3D deep CNN model to detect lesions in the breast cancer MRI dataset. They deployed a custom dataset with 1537 female patients and classify them as benign or malignant. They achieved an accuracy of $83.7 \%$ for the diagnostic task and a dice distance score of 0.501 for the detection task. The authors in [73] proposed a DL integrated architecture with the capability of performing classification, segmentation, and detection for the screening of breast masses as benign or malignant. They used digital $\mathrm{X}$-ray mammograms from the INbreast database. Their model achieved a mass detection accuracy of $98.96 \%$, while for mass segmentations, they achieved a dice score of $92.69 \%$. To augment the dataset, the authors applied rotation 8 times to synthetically increase the size of the dataset. Nasrullah et al. [74] deployed DL-based architectures for the diagnosis of malignant nodules in lung cancer. They conducted studies on LUNA16 and LIDC-IDRI datasets. They used faster region $\mathrm{CNN}$ and U-Net styled architecture to achieve an accuracy of $94.17 \%$ on the classification task. The authors in [75] used a DL-based system for screening lung cancer using CT scans. They used LIDC-IDRI and Kaggle data science bowl challenge datasets for the experiments. Their system was based on 3D CNN architectures. The authors used heavy augmentations to artificially increase the size of the datasets using methods such as rotations, scaling, translation, and reflection. They achieved a dice coefficient of 0.4 on the LIDC-IDRI dataset. Shkolyar et al. [76] deployed DL-based models for the detection of papillary and flat bladder cancer. They used CNNs to construct an image analysis platform. They used two datasets of 100 and 54 subjects. Their model successfully detected 42 of 44 papillary and flat bladder cancers. They reported a pertumor sensitivity of $90.9 \%$. Fourcade et al. [77] used a combination of DL and superpixel segmentation-based methods to segment full body organs such as the brain and heart from Positron Emission Tomography (PET) images. To synthetically increase the size of the dataset, the authors deployed rotations, scaling, mirroring, and elastic deformations. Their best performing model achieved a dice score of 0.93 . The authors in [78] deployed DL architectures to detect brain metastasis on MRI. They used data from 121 subjects in their proposed study. They used a faster region CNN model achieving an area under the ROC curve of 0.79 . Ma et al. 
TABLE 2: Summary of the studies for the prognosis/prediction of cancers.

\begin{tabular}{|c|c|c|c|c|}
\hline Publication & Type(s) of cancer & Type of data & Methods & Performance \\
\hline$[50]$ & Astrocytic tumor & Microarray gene dataset & ANN & $96.15 \%$ accuracy \\
\hline$[51]$ & Breast cancer & $\begin{array}{l}\text { Nuclear morphometric } \\
\text { features }\end{array}$ & ANNs & Good ( $>5$ years) and bad ( $<5$ years) prognoses \\
\hline$[52]$ & $\begin{array}{l}\text { Breast invasive } \\
\text { carcinoma }\end{array}$ & Gene expression data & $\begin{array}{l}\text { Multiomics neural } \\
\text { networks }\end{array}$ & Improved performance using more omics data \\
\hline$[53]$ & Breast cancer & TCGA & $\begin{array}{l}\text { Random forest, } \\
\text { neural network }\end{array}$ & Log-rank $p<0.05$ \\
\hline$[54]$ & $\begin{array}{l}\text { Malignant } \\
\text { melanoma }\end{array}$ & Custom dataset & $\begin{array}{l}\text { Nonlinear ANN } \\
\text { model }\end{array}$ & ANN model performs better than Cox model \\
\hline$[55]$ & Multiple & $\begin{array}{l}\text { WHAS, SUPPORT, } \\
\text { METABRIC, Rotterdam } \\
\text { tumor bank }\end{array}$ & $\begin{array}{l}\text { Deep feedforward } \\
\text { neural network }\end{array}$ & $\begin{array}{l}\text { Better prognostic accuracy than the clinical experts } \\
\text { for the prognosis of nasopharyngeal carcinoma }\end{array}$ \\
\hline$[56]$ & $\begin{array}{l}\text { Glioblastoma } \\
\text { multiforme }\end{array}$ & TCGA & $\begin{array}{l}\text { Pathway-associated } \\
\text { sparse deep neural } \\
\text { network }\end{array}$ & $\mathrm{AUC}=0.6622 \pm 0.013, F_{1}=0.3978 \pm 0.016$ \\
\hline$[57]$ & Breast cancer & $\begin{array}{c}\text { Gene expression } \\
\text { profile+copy number } \\
\text { alteration profile+clinical } \\
\text { data }\end{array}$ & $\begin{array}{l}\text { Multimodal deep } \\
\text { neural network }\end{array}$ & $\begin{array}{l}\text { The proposed method achieves better performance } \\
\text { than the prediction methods with single- } \\
\text { dimensional data and other existing approaches }\end{array}$ \\
\hline$[58]$ & $\begin{array}{l}\text { Hepatocellular } \\
\text { carcinoma }\end{array}$ & TCGA & DL-based model & $\begin{array}{l}p \text { value }=7.13 \times 10^{-6} \\
\text { Concordance index }=0.68\end{array}$ \\
\hline$[59]$ & Colorectal cancer & $\begin{array}{l}\text { Images of tumor tissue } \\
\text { samples }\end{array}$ & $\begin{array}{l}\text { Combined } \\
\text { convolutional and } \\
\text { recurrent } \\
\text { architectures }\end{array}$ & $\begin{array}{c}\text { Prediction with only small tissue areas } \\
\text { (hazard ratio } 2.3 \text { ), tissue microarray spot } \\
\text { (hazard ratio } 1.67 \text { ), and whole-slide level } \\
\text { (hazard ratio } 1.65 \text { ) }\end{array}$ \\
\hline$[60]$ & Ovarian cancer & CT images & $\begin{array}{l}\text { Combined DL and } \\
\text { Cox proportional } \\
\text { hazards model }\end{array}$ & Concordance index was 0.713 and 0.694 \\
\hline$[61]$ & Multiple & TCGA & ANN framework & $\begin{array}{c}\text { Same or better predictive accuracy compared to } \\
\text { other methods }\end{array}$ \\
\hline$[62]$ & Multiple & $\begin{array}{l}\text { WHAS, SUPPORT, \& } \\
\text { METABRIC }\end{array}$ & $\begin{array}{l}\text { Cox proportional } \\
\text { hazards deep neural } \\
\text { network }\end{array}$ & $\begin{array}{c}\text { Superior in predicting personalized treatment } \\
\text { recommendations }\end{array}$ \\
\hline$[63]$ & $\begin{array}{l}\text { Lower-grade glioma } \\
\text { and glioblastoma }\end{array}$ & TCGA & CNNs & Median concordance index $=0.754$ \\
\hline$[64]$ & Mesothelioma & TCGA+French source & CNNs & Concordance index of 0.656 on TCGA cohort \\
\hline$[65]$ & Multiple & $\begin{array}{l}\text { TCGA+Gene Expression } \\
\text { Omnibus dataset }\end{array}$ & DL-based model & $\begin{array}{c}\text { For both marker types, the specificity of normal } \\
\text { whole blood was } 100 \%\end{array}$ \\
\hline
\end{tabular}

[79] used you only look once v3 dense multireceptive fields $\mathrm{CNN}$ for thyroid cancer nodule detection. They used ultrasound images and deployed different data augmentation methods such as color jitter, change saturation, exposure, and hue on two datasets of 152 and 699 images. The number of images increased to 10845 after the application of data augmentation schemes. The values of mean average precision (mAP) reported by the authors were 90.05 and 95.23. Das et al. [80] proposed a system combining watershed segmentation, Gaussian mixture model (GMM), and deep neural network for the classification and segmentation of liver cancer using CT scans. Their model performed recognition of hemangioma, hepatocellular carcinoma, and metastatic carcinoma subjects. They employed 225 CT scans in their study achieving a dice score of 0.9743 on the testing set for the segmentation task and an accuracy of $99.38 \%$ for the multiclass classification task. The authors in [81] proposed a DL-based model for the segmentation of histopathology images of the liver organ. Their proposed DL model combined residual block, bottleneck block, and an attention decoder block. The authors further created a new dataset of 80 histopathology images which they named as the KMC liver dataset and proposed a joint loss function combining dice and Jaccard losses. They conducted their experiments on two datasets: KMC liver and multiorgan Kumar datasets. Each image in the Kumar dataset has a dimension of $1000 \times 1000$ while each image in the KMC liver dataset has a dimension of $1920 \times 1440$. Their model achieved a Jaccard index of 0.7206 on the KMC liver dataset and 0.6888 on the Kumar dataset. Wang and Chung [82] proposed a modified U-Net-based architecture for the segmentation and diagnosis of the colon gland. The authors employed two 
datasets for the experiments: the gland segmentation dataset from Medical Image Computing and Computer-Assisted Intervention (MICCAI) challenge and an independent colorectal adenocarcinoma gland dataset. The authors conducted validation experiments on 378 images augmented using elastic transformation, cropping, rotation, flipping, blurring, and distortion operations. Their model achieved a dice score of 0.929 on the gland segmentation dataset from MICCAI and 0.89 on the independent colorectal adenocarcinoma gland dataset for the segmentation task. The authors in [83] proposed a CNN architecture combining three CNNs for the segmentation and classification of colorectal cancer using MRI images of 28 adenocarcinomas and 5 mucinous carcinomas. Their model achieved a dice score of 0.60 , precision of 0.76 , and recall of 0.55 on the testing set. The authors used cropping and min-max scaling as preprocessing methods. Juebin et al. [84] proposed segmentation algorithms based on U-Net models from ultrasound images of ovarian cancer. Image clipping was used as a preprocessing method. The methods are validated on 127 patients and a total of 469 images. Their best model achieved a dice score of 0.87, an average Pearson correlation of 0.90 , and an average intraclass correlation of 0.89 . Shibata et al. [85] used the mask R-CNN algorithm for the segmentation of 1208 healthy and 533 gastric cancer endoscopic images. The resolution of the images ranges from $640 \times 480$ to $1440 \times 1080$ pixels. Their model achieved an average dice index of $71 \%$. Wang and Liu [86] proposed an architecture based on Deeplab v3+ for the segmentation of 1340 pathological slices of gastric cancer. The authors used image mirroring, random flip, scale, and rotation as augmentation techniques. Mean subtraction followed by division with variance was used as a preprocessing method. Their model achieved a dice score of 0.9166. Shrestha et al. [87] proposed a DL system combining four U-Net models. They used MRI images of prostate cancer from an online database. Each image has a resolution of $256 * 256$ pixels. They used a combination of modified dice and binary crossentropy loss for the segmentation task. They preprocess the images using denoising and intensity normalization procedures achieving an overall average accuracy of 95.3\%. Liu et al. [88] proposed a DL method integrating mask R-CNN and Inception version 3 models for the classification, segmentation, and detection tasks of prostate cancer. They used a dataset of 1200 ultrasound images. Their model achieved a dice score of 0.88 and a precision of $76 \%$ on malignant and $75 \%$ on benign classes for the classification task using an Inception v3 architecture. The authors in [89] proposed a 2D U-Net model deploying CT images of 556 cases of prostate cancer. They achieved a dice score of $0.85,0.94$, and 0.85 for three organs, namely, prostate, bladder, and rectum, respectively. Liang et al. [90] developed a DL-based model employing CNN architecture for the segmentation of pancreatic tumors. The authors deployed a dataset of T1w MRI images of 40 subjects. They achieved a dice score of 0.73 using rotation and flipping as data augmentation methods. The authors in [91] proposed a DL method using spiral transformation to perform segmentation of MRI images of pancreatic can- cer. The authors used rotation and spiral transformation as data augmentation methods. They deployed a dataset of MRI images belonging to 73 patients. Their architecture is a combination of ResNet and U-Net architectures. They achieved a dice score of $0.656 \pm 0.1021$.

Table 3 displays a summary of the studies for the task of segmentation and detection of cancers covered in this subsection.

4.3. Classification of Breast Cancer. Huynh et al. [92] used DL methods to classify regions of interest taken from ultrasound images. Cystic, benign, or malignant labels were assigned to each region. Two binary classification tasks were performed using pretrained CNNs, nonmalignant (benign+cystic)/malignant and benign/malignant. They used SVM as a classifier on the $\mathrm{CNN}$-derived features. On the nonmalignant/malignant classification task, they obtained an AUC of 0.9 while on the benign/malignant task, their method obtained an AUC of 0.88. The authors in [93] used CNNs as their DL approaches and introduced the concept of a matching layer to convert grayscale to red, green, and blue patterns. They used 882 ultrasound images obtained from two publicly available datasets. Using fine-tuning and matching layer, their method approached an AUC of 0.936 on a test set of 150 cases. Byra et al. [94] used DL transfer learning-based approaches such as Inception version 3 and VGG19 architectures on reconstructed B-mode images experiencing a decrease in classification performances. To counter this, they used data augmentation to reconstruct B-mode images achieving better performances on breast ultrasound images. The authors in [95] combined crossmodal and cross-domain transfer learning for the benign/malignant classification task. In comparison to training from scratch and simple fine-tuning, their approach achieved better performance with $97 \%$ accuracy on ultrasound images. Hadad et al. [96] deployed cross-modal transfer learning using mammography images achieving an accuracy of 0.93 which is better than cross-domain transfer learning. The authors in [97] presented a study on the use of MRI in screening individuals younger than 40 years confirming the effectiveness of MRI as a modality of choice for such diagnoses. They reported a very high sensitivity around $93 \%$ to $100 \%$ and low specificity in the range of $37 \%$ to $97 \%$. They found MRI to be effective especially after reconstructive surgery. $\mathrm{Hu}$ et al. [98] developed a transfer learning methodology using an MRI modality with multiple parameters. They used different sequences such as dynamic contrast-enhanced and a T2-weighted sequence to distinguish benign lesions from malignant. They used image, feature, and classifier fusion methods and achieved an AUC of 0.87 for the feature fusion scheme that statistically outperformed other methods. The authors in [99] proposed a methodology using Inception version 4 and the residual network transfer learning architectures as well as a recurrent CNN architecture on the 2015 Breast Cancer Classification Challenge and BreakHis datasets for binary and multiclass classification tasks. They used rotation, translation, and other data augmentation methods to artificially increase the size of the datasets achieving an accuracy of $97.57 \pm 0.89 \%$ on multiclass and 
TABLE 3: Summary of the studies for the segmentation/detection of cancers.

\begin{tabular}{|c|c|c|c|}
\hline Publication & Type(s) of cancer & Type of data & Methods \\
\hline$[66]$ & Breast cancer & $\begin{array}{l}\text { Ultrasound images, } 2 \text { datasets } \\
\text { (A \& B })\end{array}$ & LeNet, U-Net, AlexNet \\
\hline$[67]$ & Breast lesions & Two custom datasets & $\begin{array}{l}\text { FCN-AlexNet, FCN-32s, FCN-16s, } \\
\text { and FCN-8s }\end{array}$ \\
\hline [68] & Breast cancer & Camelyon 16 & DL algorithm \\
\hline [69] & Breast cancer & $\begin{array}{l}\text { International Conference on } \\
\text { Pattern Recognition 2012, } \\
\text { MITOS-ATYPIA-14, Tumor } \\
\text { Proliferation Assessment } \\
\text { Challenge } 2016\end{array}$ & $\begin{array}{l}\text { Faster region CNN, ResNET-50, } \\
\text { DenseNet-201 }\end{array}$ \\
\hline$[70]$ & Multiple & $\begin{array}{c}\text { Custom histopathology image } \\
\text { dataset }\end{array}$ & $\begin{array}{l}\text { Multilayer perceptron, logistic } \\
\text { modal tree, sequential minimal } \\
\text { optimization, Naïve Bayes, random } \\
\text { forest, rotation forest, J-Rip, and } \\
\text { PART algorithms }\end{array}$ \\
\hline$[71]$ & Breast cancer & $\begin{array}{c}\text { ICPR } 2014 \text { mitosis dataset, } \\
\text { TUPAC } 2016 \text { mitotic cell } \\
\text { dataset }\end{array}$ & Modified regional CNN \\
\hline$[72]$ & Breast cancer & $\begin{array}{l}\text { Custom dynamic contrast- } \\
\text { enhanced MRI dataset }\end{array}$ & 3D deep $\mathrm{CNN}$ architecture \\
\hline$[73]$ & Breast cancer & INbreast database & Different DL methods \\
\hline
\end{tabular}

[74] Lung nodules

\section{LUNA16, LIDC-IDRI}

Lung cancer

Bladder cancer

Full body

Brain metastases

Thyroid nodules

Liver cancer

Liver cancer

Colorectal cancer

MICCAI gland segmentation dataset, colorectal adenocarcinoma gland dataset

Custom dataset of MRI images Colorectal cancer of 28 adenocarcinomas and 5 mucinous carcinomas
Two deep 3D customized mixed link network encoder-decoder architectures

3D CNN architectures

DL algorithm

DL-based approach

DL-based approach (faster regionbased CNN model)

You only look once v3 dense multireceptive field CNN

Watershed segmentation, Gaussian mixture model (GMM), and deep neural network

DL model combining residual

block, bottleneck block, and attention decoder

Modified U-Net-based architecture

$\mathrm{CNN}$ architecture which is a combination of three $\mathrm{CNN}$ architectures
Rotation forest algorithm achieved an accuracy of $85.7 \%$ for binary classification between cancerous and noncancerous cells

Precision $=0.76$, recall $=0.72, F_{1^{-}}$ score $=0.736$ on TUPAC 2016 dataset

$83.7 \%$ accuracy, $90.8 \%$ sensitivity, $69.3 \%$ specificity, AUC of 0.859 , overall dice distance of $0.501 \pm$

$$
0.274
$$

Accuracy of $98.96 \%$, MCC of $97.62 \%, F_{1}$-score of $99.24 \%$, Jaccard similarity coefficient of $86.37 \%$

\section{Accuracy of $94.17 \%$}

Dice coefficient for LIDC-IDRI of 0.40 , with 0.25 precision and 0.93 recall

Per-frame sensitivity and specificity were $90.9 \%$ and $98.6 \%$

Dice score of $0.93 \pm 0.05$

$96 \%$ sensitivity, $\mathrm{AUC}=0.79$

$\mathrm{mAP}=90.05$ and 95.23

Dice score of 0.9743 , accuracy of $99.38 \%$

Jaccard index of 0.7206 on KMC liver dataset and 0.6888 on Kumar dataset

Dice score of 0.929 on MICCAI gland segmentation dataset, 0.89 on the colorectal adenocarcinoma gland dataset

Dice score of 0.60 , precision of 0.76 , and recall of 0.55 
TABLE 3: Continued.

\begin{tabular}{|c|c|c|c|c|}
\hline Publication & Type(s) of cancer & Type of data & Methods & Performance \\
\hline$[84]$ & Ovarian cancer & $\begin{array}{l}\text { Custom dataset of } 127 \text { patients } \\
\text { and a total of } 469 \text { images }\end{array}$ & U-Net models & $\begin{array}{c}\text { Dice score of } 0.87 \text {, an average } \\
\text { Pearson correlation of } 0.90 \text {, and an } \\
\text { average intraclass correlation of } \\
0.89\end{array}$ \\
\hline$[85]$ & Gastric cancer & $\begin{array}{l}\text { Custom dataset of } 1208 \text { healthy } \\
\text { and } 533 \text { endoscopic images }\end{array}$ & Mask R-CNN algorithm & Average dice index of $71 \%$ \\
\hline$[86]$ & Gastric cancer & $\begin{array}{l}\text { Custom dataset of } 1340 \\
\text { pathological slices }\end{array}$ & Deeplab v3+ & Dice score of 0.9166 \\
\hline$[87]$ & Prostate cancer & $\begin{array}{l}\text { MRI images from an online } \\
\text { database }\end{array}$ & $\begin{array}{l}\text { DL system combining four U-Net } \\
\text { models }\end{array}$ & Overall average accuracy of $95.3 \%$ \\
\hline$[88]$ & Prostate cancer & $\begin{array}{l}\text { Custom dataset of } 1200 \\
\text { ultrasound images }\end{array}$ & $\begin{array}{l}\text { DL method integrating mask } \\
\text { R-CNN and Inception version } \\
3 \text { models }\end{array}$ & $\begin{array}{c}\text { Dice score of } 0.88 \text {, a precision of } \\
76 \% \text { on malignant and } 75 \% \text { on } \\
\text { benign classes for the classification } \\
\text { task using an Inception v3 } \\
\text { architecture }\end{array}$ \\
\hline [89] & Prostate cancer & $\begin{array}{l}\text { Custom dataset of CT images of } \\
556 \text { cases }\end{array}$ & 2D U-Net model & $\begin{array}{l}\text { Dice score of } 0.85,0.94 \text {, and } 0.85 \\
\text { for prostate, bladder, and rectum, } \\
\text { respectively }\end{array}$ \\
\hline$[90]$ & Pancreatic cancer & $\begin{array}{l}\text { Custom dataset of T1w MRI } \\
\text { images of } 40 \text { subjects }\end{array}$ & $\mathrm{CNN}$ architecture & Dice score of 0.73 \\
\hline$[91]$ & Pancreatic cancer & $\begin{array}{l}\text { Custom dataset of MRI images } \\
\text { belonging to } 73 \text { patients }\end{array}$ & $\begin{array}{l}\text { DL method using spiral } \\
\text { transformation }\end{array}$ & Dice score of $0.656 \pm 0.1021$ \\
\hline
\end{tabular}

an accuracy of $97.95 \pm 1.07 \%$ on binary classification tasks. Bayramoglu et al. [100] used single and multitask CNN architectures to predict malignancy and image magnification levels. Cropping and rotation on the BreakHis dataset were deployed to augment the dataset. They achieved a classification rate of $83.72 \%$ for the benign/malignant binary classification task using a single task and an accuracy of $82.13 \%$ using multitask CNN architecture. The authors in [101] proposed an approach for progressive combining of weak DL classifiers into a stronger classifier for carcinomas/noncarcinomas binary and normal/benign/in situ/invasive carcinomas multiclass (4 classes) classification tasks. They used BreakHis and 2015 bioimaging breast histology classification challenge datasets. They deployed augmentation methods such as reflection, random cropping, rotation, and translation of an image. They achieved a classification accuracy of 99.5\% and $96.9 \%$ for binary classification tasks using the 2015 bioimaging breast histology classification challenge database and BreakHis database while for multiclass classification, they achieved $96.4 \%$ classification accuracy on the 2015 bioimaging breast histology classification challenge database. Kassani et al. [102] used an ensemble of transfer learning architectures for binary classification of breast cancer. They used VGG19, MobileNet, and DenseNet architectures on four benchmark datasets: BreakHis, PatchCamelyon, 2015 Bioimaging challenge, and 2018 ICIAR datasets. They used flipping, zoom, shear, rotation, etc., as data augmentation methods. They achieved accuracies of $98.13 \%, 94.64 \%, 95 \%$, and $83.1 \%$ on these datasets. The authors in [103] proposed a DL method for multiclass (8 classes) classification of histopathological images on the BreakHis dataset. Data augmentation methods such as rotation, flipping, sharing, and their combinations were deployed to achieve a correct classification rate of $95.48 \%$ on the multiclass classification task. Toğaçar et al. [104] deployed a DL model for multiclass (8 classes) classification of breast histopathological images on the BreakHis dataset. They used convolutional, attention, residual, pooling, and dense blocks along with hypercolumn technique to build their architecture. As data augmentation methods, they used flipping, shifting, change of brightness, and rotation achieving $98.51 \%$ accuracy. The authors in [105] used a combination of DenseNet and Xception transfer learning architectures for benign/malignant binary and magnification-specific multiclass classification tasks. They used the BreakHis dataset achieving an accuracy of $99 \%$ and $92 \%$ on binary and multiclass classification tasks, respectively, while deploying stain normalization for preprocessing of images. Spanhol et al. [106] performed experiments for the binary (benign/malignant tumors) classification task using histology images. They report an accuracy of $90.0 \pm 6.7 \%$ on images obtained from the BreakHis dataset. The authors in [107] proposed a DL model for the multiclass (8 classes) classification task using histopathological images. They conducted experiments on the BreakHis dataset. For data augmentation, the authors deployed rotation, level/vertical flipping, translation techniques, etc., and their combinations achieving a patient-level accuracy of $94.7 \pm 3.6 \%$. Bardou et al. [108] compared CNNs and traditional machine learning techniques such as bag of words and linear coding using SVMs. They deployed BreakHis datasets for both binary and multiclass (8 classes) classification tasks to categorize images into benign/malignant classes and their subclasses. The authors achieved accuracies of $98.33 \%$ and $88.23 \%$ for binary and multiclass classifications, respectively, using the deployed approaches. The authors in [109] combined four residual networks for binary (benign/malignant) 
and multiclass classification of histology images using the BreakHis dataset. They achieved an accuracy of $96.25 \%$ for the eight-class classification task. The authors deployed rotation, flipping, translation, and color variation augmentation as data enhancing methods while stain normalization as a preprocessing method. Budak et al. [110] used a DL model combining FCN and bi-LSTM architectures on the BreakHis dataset for binary (benign/malignant) classification achieving an accuracy of $96.32 \%$. The authors in [111] used a DLbased model for binary (benign/malignant) classification of histopathological images. They performed experiments on the BreakHis dataset fusing ResNet-18, ResNet-50, and AlexNet architectures using belief theory. They achieved an image-level accuracy of $96.88 \%$. Sudharshan et al. [112] deployed a weakly supervised scheme for the binary classification of benign and malignant tumors using histopathology images. They deployed the BreakHis dataset achieving an accuracy of $92.1 \%$ at $40 \mathrm{x}$ magnification. An important contribution of their approach is the absence of the need for labelling the images. The authors in [38] deployed CNNs for both binary (carcinoma/noncarcinoma) and multiclass (normal/benign/in situ/invasive) classification tasks. They used the 2015 Bioimaging breast histology classification challenge dataset in their study. Their architectures were able to retrieve information at different scales. For the multiclass classification task, the authors achieved an accuracy of $77.8 \%$ while for the binary classification task, they achieved an accuracy of $83.3 \%$ using rotation and mirroring as data enhancement methods for both these tasks. Rakhlin et al. [113] deployed different transfer learning architectures using microscopic histological images from the ICIAR 2018 Grand Challenge dataset for binary (carcinomas/noncarcinomas) and multiclass (four classes) classification tasks. They used pretrained ResNet-50, Inception version 3, and VGG16 architectures. They deployed normalization, downscaling, cropping, and color variation as augmentation schemes achieving a correct classification rate of $87.2 \%$ for multiclass classification and $93.8 \%$ for the binary classification task. The authors in [114] extracted smaller/larger patches using a clustering approach and a CNN (ResNet50 architecture) at cell and tissue levels deploying the 2015 Bioimaging breast histology classification challenge dataset. For the multiclass ( 4 classes) classification task, the authors reported accuracy of $88.89 \%$ using the proposed approach. The authors deployed stain normalization procedure as a preprocessing method. Shallu and Mehra [115] demonstrated the use of three different transfer learning architectures such as VGG16, VGG19, and ResNet-50 for the classification of histological images on the BreakHis dataset. They deployed rotation as the data enhancement scheme. They found the performance of a fine-tuned VGG16 with logistic regression classifier to be the best achieving an accuracy of $92.6 \%$ with this classifier. The authors in [116] deployed CNN, $K$ nearest neighbour (KNN), Inception version 3, SVM, and ANN algorithms for the binary (benign/malignant) classification task. They used different schemes for preprocessing and data enhancement such as gray scaling, channel standardization, flipping, rotation, and cropping as well as image segmentation to reach an accuracy of $97 \%$ using ANN architecture. Bevilacqua et al. [117] evaluated two different frameworks for binary and multiclass classification of irregular/regular/stellar/no opacity lesions from segmented high-resolution images. They used ANN classifiers with hand-crafted and morphological features for the first framework. For the second framework, they used different CNN models especially a VGG model. They reported accuracy of $84.19 \%$ for the first framework on binary and $74.84 \%$ on multiclass classification tasks while for the second framework, they obtained an accuracy of $92.02 \%$ for binary and multiclass classification tasks. The authors in [118] make a contrast between two machine learning approaches for the multiclass (8 classes) classification task using histopathological images on the BreakHis dataset. The first approach used handcrafted features while the second approach used CNN as a feature extractor. They used VGG16, VGG19, and ResNet-50 as their CNN models. They used rotation, translation, scaling, and flipping as data enhancement methods. The VGG16 model reaches an accuracy of $93.25 \%$ at the patient level for the multiclass classification task. Spanhol et al. [119] proposed a DL model that reused a previously trained CNN model on the BreakHis dataset achieving an $F_{1}$-score of 90.3 at the subject level. The authors in [120] exploited global covariance information using a matrix power normalization procedure into a simple CNN model. This arrangement can exploit second-order statistical information producing effective representations from histological images. On the BreakHis dataset for the binary (benign/malignant) classification task, they achieved an accuracy of $97.92 \%$ at the subject level while employing cropping and flipping operations to enhance the size of the dataset synthetically. Khan et al. [121] used different transfer learning (GoogLeNet, VGGNet, and ResNet) architectures for binary classification of benign/malignant tumor cells while deploying BreakHis and a custom dataset. For data augmentation, scaling, rotation, translation, and color augmentation methods were used by them to achieve a correct classification rate of $97.67 \%$. The authors in [122] introduced an information-based architecture that is designed to exploit clinical information. There are six types of records in their dataset of 100 subjects, such as encounter notes, operation records, pathology notes, radiology notes, progress notes, and discharge summaries. They used fine-tuned transformer models from pretrained bidirectional encoder representations achieving a precision of 0.976 for relation recognition. Naik et al. [123] deployed a DL model to assess estrogen status from whole-slide histopathological images. They used the Australian Breast Cancer Tissue Bank as well as TCGA datasets in their study and further deployed flipping, rotation, color jitter, and cutout regularization as augmentation methods. Their model achieved an AUC of 0.861 on TCGA and an AUC of 0.905 on Australian Breast Cancer Tissue Bank datasets. The authors in [124] compared different DL techniques for the classification of mammograms. They used single as well as 4-model averaging to conduct their experiments on INbreast as well as an independent database. They used different data enhancement techniques such as flipping, rotation, intensity shifting, and zoom. The single model achieved an AUC of 0.88 while 4-model averaging achieved an AUC of 0.91 on the independent database. On the INbreast dataset, the single model achieved an AUC of 
0.95 while 4-model averaging achieved an AUC of 0.98 . Their study shows the superiority of combining models over a single model.

Table 4 displays a summary of the studies for the classification of breast cancer covered in this subsection.

4.4. Classification of Colorectal Cancer, Gastric Cancer, Bladder Cancer, Lung Cancer, Prostate Cancer, Skin Cancer, Liver Cancer, Head and Neck Cancer, Pancreatic Cancer, and Other Types of Cancers. Kather et al. [125] deployed different transfer learning architectures for multiclass (9 classes) classification of colorectal cancer subjects. They used VGG19, AlexNet, SqueezeNet, GoogLeNet, and ResNet-50 models on two datasets of 86 and 25 subjects reaching an accuracy of $98.7 \%$ and greater than $94 \%$ on them. The authors in [126] deployed a CNN architecture to extract features from Optical Coherence Tomography (OCT) images of colorectal cancer subjects. Their network was trained using 26000 OCT images representing 42 areas achieving an AUC of 0.998. Dong et al. [127] deployed a DL method to exploit information in multiphase CT nomograms in gastric cancer subjects. They used three cohorts to test the effectiveness of their model achieving a discrimination rate of $0.821,0.797$, and 0.822 in the primary, external validation, and international validation cohorts. Woerl et al. [128] deployed a DL method to identify bladder cancer from histomorphological images. They used 2 datasets of 407 and 16 subjects each from TCGA and custom cohorts, respectively, achieving accuracies of $69.91 \%$ and $75 \%$ on TCGA and custom subsets, respectively. Wang et al. [129] used the idea of weakly supervised learning exploiting image-level labels for the classification of lung cancer images. They used two datasets, one from TCGA and the other is a custom dataset. To enhance the training set, color jittering, translation, flipping, and rotation were used.

Their model successfully achieves an accuracy of $97.3 \%$ and an AUC of $85.6 \%$ on custom and TCGA datasets. Karimi et al. [130] used a DL method combining three separate CNNs that used different patch sizes for the classification of histopathological images with limited data. They used new data enhancement methods such as elastic deformation and augmentation in the space of learned features for binary classification of cancerous/benign and lowgrade-high-grade patches achieving an accuracy of $92 \%$ and $86 \%$, respectively, on both binary classification tasks. Dascalu and David [131] used DL architectures for binary classification of benign/malignant cases of skin cancer subjects. They used a skin magnifier with polarized light and an advanced dermoscope to construct their datasets. The authors achieved an F2-score sensitivity of $91.7 \%$ and $89.5 \%$ respectively for skin magnifier with polarized light and advanced dermoscope images. The authors in [132] used DL techniques to build a skin cancer classification model for binary and multiclass classification of malignant and benign skin tumors. They used Kaohsiung Chang Gung Memorial Hospital and HAM10000 datasets in their study. Their model achieved an accuracy of $85.8 \%$ on the HAM10000 dataset for 7-class classification tasks. On the Kaohsiung Chang Gung Memorial Hospital dataset, their model achieved an accuracy of $72.1 \%$ for 5 -class classification and $89.5 \%$ for binary classification tasks. Thomas et al. [133] applied interpretable DL models to classify skin cancers in a histopathological setting. They studied three types of cancers basal cell carcinoma, squamous cell carcinoma, and intraepidermal carcinoma. They deployed a multiclass (12 classes) classification model to achieve accuracies between $93.6 \%$ and $97.9 \%$. To solve the class imbalance problem and to increase the size of the dataset, they used flipping and rotation as data augmentation methods to increase the size of the dataset 8 times. The authors in [134] developed a CNN model for the classification of melanoma and nevi. They used a dataset of 11444 images belonging to five categories. They deployed novel DL techniques to train a single $\mathrm{CNN}$ model. In addition, they also asked 112 dermatologists to grade the images. Then, they used a gradient boosting method to develop a new classifier for binary (benign/malignant) and multiclass (5 classes) classification tasks achieving accuracies of $86.5 \%$ and $82.95 \%$ on both these tasks. Sun et al. [135] developed a DL method to classify liver cancer subjects as abnormal/normal on publically available TCGA datasets. Transfer learning and multiple instance learning were combined for the classification of patch features. The authors used tissue extraction, color normalization, and patch extraction for preprocessing of histopathological images. Diao et al. [136] used a transfer learning-based $\mathrm{CNN}$ architecture named Inception version 3 to classify nasopharyngeal carcinoma subjects into three classes. They used a total of 1970 images of 731 subjects. The three classes considered in their study were chronic nasopharyngeal inflammation, lymphoid hyperplasia, and nasopharyngeal carcinoma. Their model achieved a mean AUC of 0.936. Liu et al. [137] used a $\mathrm{CNN}$ classifier to diagnose subjects with pancreatic cancer using contrast-enhanced CT images. They used three different datasets to test the effectiveness of their approach. The first dataset named local test set 1 has 295 patients with pancreatic cancer and 256 controls for training and 75 patients with pancreatic cancer and 64 controls for validation. The second dataset named local test set 2 has 101 patients with pancreatic cancers and 88 controls while the third dataset named the US dataset has 281 pancreatic cancer subjects and 82 controls. In local test set 1 , local test set 2 , and US datasets, their model achieved an accuracy of $98.6 \%$, $98.9 \%$, and $83.2 \%$, respectively. To augment the datasets, the authors used moving window and flipping operations. Korfiatis et al. [138] compared the performances of ResNet-18, ResNet-34, and ResNet-50 architectures for the classification of MRI scans of 155 subjects for multiclass (3 classes) classification of no tumor, methylated methylguanine methyltransferase methylation, or nonmethylated classes. ResNet-50 architecture achieved the best performance with an accuracy of 94.9\%; ResNet-34 architecture achieved an accuracy of $80.72 \%$ while ResNet-18 architecture achieved an accuracy of $76.75 \%$. The authors in [139] used a two-phase training to study and mitigate class biasedness using a DL-based CNN model for the classification of breast cancer histological images. They conducted their experiments using MITOS12 and 2016 Tumor Proliferation 
TABLE 4: Summary of the studies for the classification of breast cancer.

\begin{tabular}{|c|c|c|c|}
\hline Publication & Type of data & Methods & Performance \\
\hline$[92]$ & Custom dataset of ultrasound images & Pretrained CNNs & $\begin{array}{c}\text { AUC of } 0.90 \text { (nonmalignant vs. malignant), } \\
\text { AUC of } 0.88 \text { (benign vs. malignant) }\end{array}$ \\
\hline [93] & $\begin{array}{l}1 \text { custom }+2 \text { publically available } \\
\text { datasets }\end{array}$ & $\begin{array}{l}\text { DL-based approach using } \\
\text { a matching layer }\end{array}$ & $\begin{array}{c}\text { AUC }=0.936 \text { (custom dataset), AUCs around } \\
0.89 \text { (publically available datasets) }\end{array}$ \\
\hline [94] & Custom dataset of ultrasound images & Inception v3, VGG19 & Robust and efficient classification performances \\
\hline [95] & Custom dataset of ultrasound images & $\begin{array}{l}\text { Training from scratch, } \\
\text { pretrained VGG16, fine- } \\
\quad \text { tuning approach }\end{array}$ & 0.97 accuracy, 0.98 AUC using fine-tuning approach \\
\hline [96] & Custom dataset of breast MRI images & $\begin{array}{l}\text { Cross-modal transfer } \\
\text { learning approach }\end{array}$ & Overall accuracy of 0.93 using cross-modal approach \\
\hline [97] & Custom dataset of breast MRI images & DL-based method & High sensitivity in the range of $93-100 \%$ \\
\hline [98] & $\begin{array}{l}\text { Custom dataset of multiparametric } \\
\text { MRI images }\end{array}$ & $\begin{array}{l}\text { Pretrained } \mathrm{CNN} \\
\text { architectures }\end{array}$ & $\mathrm{AUC}_{\text {feature fusion }}=0.87$ \\
\hline [99] & $\begin{array}{c}\text { BreakHis, Breast Cancer Classification } \\
\text { Challenge } 2015\end{array}$ & $\begin{array}{l}\text { Inception recurrent } \\
\text { residual CNN model }\end{array}$ & $\begin{array}{l}100 \% \text { for the binary and multiclass (Breast Cancer } \\
\text { Classification Challenge } 2015 \text { dataset) }\end{array}$ \\
\hline$[100]$ & BreakHis & $\begin{array}{l}\text { Single-task CNN, } \\
\text { multitask CNN }\end{array}$ & $\begin{array}{c}\text { Patient score of } 83.72 \% \text { for binary classification using } \\
\text { single-task CNN }\end{array}$ \\
\hline$[101]$ & $\begin{array}{l}2015 \text { bioimaging breast histology } \\
\text { classification challenge, BreakHis } \\
\text { dataset }\end{array}$ & $\begin{array}{l}\text { Progressive DL-based } \\
\text { models }\end{array}$ & $\begin{array}{c}\text { Recognition rate of } 96.4 \% \text { and } 99.5 \% \text { on multiclass and } \\
\text { binary classification tasks on } 2015 \text { bioimaging breast } \\
\text { histology classification challenge }\end{array}$ \\
\hline$[102]$ & $\begin{array}{l}\text { BreakHis dataset, PatchCamelyon } \\
\text { dataset, } 2015 \text { Bioimaging challenge } \\
\text { dataset, } 2018 \text { ICIAR dataset }\end{array}$ & $\begin{array}{l}\text { VGG19, MobileNet, } \\
\text { DenseNet }\end{array}$ & Accuracy of $98.13 \%$ on BreakHis dataset \\
\hline$[103]$ & BreakHis dataset & $\begin{array}{l}\text { DL and hierarchical } \\
\text { classification approach }\end{array}$ & Accuracy of $95.48 \%$ on the multiclass classification task \\
\hline$[104]$ & BreakHis dataset & Integrated DL model & $\begin{array}{l}\text { 98.51\% classification success on the multiclass } \\
\text { classification task }\end{array}$ \\
\hline$[105]$ & BreakHis dataset & $\begin{array}{l}\text { DenseNet and Xception } \\
\text { architectures }\end{array}$ & $\begin{array}{c}99 \% \text { and } 92 \% \text { accuracy on binary and multiclass } \\
\text { classification tasks }\end{array}$ \\
\hline [106] & BreakHis dataset & DL-based model & $\begin{array}{c}\text { Mean recognition rate of } 90.0 \pm 6.7 \text { for binary } \\
\text { classification }\end{array}$ \\
\hline$[107]$ & BreakHis dataset & DL-based model & Accuracy of $94.7 \pm 3.6$ for multiclass classification \\
\hline [108] & BreakHis dataset & $\begin{array}{l}\text { Bag of words, locality- } \\
\text { constrained linear coding, } \\
\text { CNNs }\end{array}$ & $\begin{array}{c}\text { For CNN model accuracies between } 96.15 \% \text { and } \\
98.33 \% \text { for the binary classification and } 83.31 \% \text { and } \\
88.23 \% \text { for the multiclass classification }\end{array}$ \\
\hline [109] & BreakHis dataset & $\begin{array}{l}\text { Combination of } 4 \text { residual } \\
\text { networks }\end{array}$ & $\begin{array}{l}\text { Correct classification rate of } 96.25 \% \text { for } 8 \text {-class } \\
\text { categorization }\end{array}$ \\
\hline$[110]$ & BreakHis dataset & $\begin{array}{l}\text { End-to-end model based } \\
\text { on FCN and bidirectional } \\
\text { LSTM }\end{array}$ & Accuracy of $96.32 \pm 0.51$ on binary classification task \\
\hline [111] & BreakHis dataset & $\begin{array}{l}\text { ResNet-18, ResNet-50, } \\
\text { and AlexNet }\end{array}$ & Image-level accuracy of $96.88 \%$ for binary classification \\
\hline$[112]$ & BreakHis dataset & $\begin{array}{l}\text { Weakly supervised } \\
\text { learning framework }\end{array}$ & $\begin{array}{c}\text { Classification rate of up to } 92.1 \% \text { for binary } \\
\text { classification }\end{array}$ \\
\hline [38] & 2015 Bioimaging challenge dataset & CNN models & $\begin{array}{l}\text { Accuracies of } 77.8 \% \text { for four classes and } 83.3 \% \text { for } \\
\text { carcinoma/noncarcinoma were achieved }\end{array}$ \\
\hline$[113]$ & ICIAR 2018 Grand Challenge & $\begin{array}{l}\text { Pretrained ResNet-50, } \\
\text { Inception v3, and VGG16 } \\
\text { architectures }\end{array}$ & $\begin{array}{c}\text { Accuracies of } 87.2 \% \text { for multiclass, } 93.8 \% \text { for binary } \\
\text { classification tasks }\end{array}$ \\
\hline$[114]$ & 2015 Bioimaging challenge database & $\begin{array}{l}\text { Clustering algorithm and } \\
\text { ResNet-50 architecture }\end{array}$ & $\begin{array}{c}88.89 \% \text { accuracy on the overall test set for multiclass } \\
\text { classification }\end{array}$ \\
\hline [115] & BreakHis dataset & & $92.60 \%$ accuracy \\
\hline
\end{tabular}


TABLE 4: Continued.

\begin{tabular}{|c|c|c|c|}
\hline Publication & Type of data & Methods & Performance \\
\hline & & $\begin{array}{l}\text { VGG16, VGG19, and } \\
\text { ResNet-50 architectures }\end{array}$ & \\
\hline$[116]$ & Custom dataset & $\begin{array}{l}\text { CNN, KNN, Inception v3, } \\
\text { SVM, and ANN models }\end{array}$ & $\begin{array}{c}\text { Accuracy of } 97 \% \text { using ANN algorithm for binary } \\
\text { classification }\end{array}$ \\
\hline$[117]$ & Custom dataset & CNN and ANN models & $\begin{array}{c}\text { Accuracy of } 92.02 \pm 0.51 \% \text { for the binary classification } \\
\text { task using a VGG model, accuracy of } 92.02 \pm 0.48 \% \text { for } \\
\text { the multiclass classification task }\end{array}$ \\
\hline$[118]$ & BreakHis dataset & $\begin{array}{l}\text { VGG16, VGG19, ResNet- } \\
50 \text { architectures }\end{array}$ & Accuracy of $93.25 \%$ for multiclass classification task \\
\hline [119] & BreakHis dataset & DL-based model & $F_{1}$-score of 90.3 \\
\hline$[120]$ & BreakHis dataset & $\begin{array}{l}\text { Deep second-order } \\
\text { pooling network }\end{array}$ & Accuracy of $97.92 \%$ for binary classification \\
\hline$[121]$ & BreakHis+custom datasets & $\begin{array}{l}\text { Pretrained CNN } \\
\text { architectures (GoogLeNet, } \\
\text { VGGNet, and ResNet) }\end{array}$ & Accuracy of $97.67 \%$ for binary classification \\
\hline$[122]$ & Custom dataset & Transformer models & Precision of 0.976 for relation recognition \\
\hline$[123]$ & $\begin{array}{c}\text { Australian Breast Cancer Tissue Bank, } \\
\text { TCGA dataset }\end{array}$ & Deep neural network & $\begin{array}{c}\text { AUC on TCGA of 0.861, AUC on Australian Breast } \\
\text { Cancer Tissue Bank was } 0.905\end{array}$ \\
\hline [124] & INbreast database & DL models & $\mathrm{AUC}=0.98$ \\
\hline
\end{tabular}

Assessment Challenge datasets. Prior to phase 1 of training, segmentation using the global binary thresholding method was applied. In phase 1 , a CNN was trained on the segmented patches using rotation and flipping data augmentation methods as well as the blue ratio histogram-based $k$-means clustering approach. In phase 2 , the dataset was again modified to reduce the effects of class imbalance yielding an F-measure of 0.79. Campanella et al. [140] proposed a DL-based system utilizing information from multiple instances in order to help the pathologists exclude information without compromising performance metrics. They used 44732 whole-slide images belonging to 15187 patients. They achieved AUC above 0.98 and $100 \%$ sensitivity for prostate cancer, basal cell carcinoma, and breast cancer metastases to axillary lymph nodes. The authors in [141] proposed two DL-based systems to detect myeloid leukemia from the leukemia microarray genetic dataset. The first DL system is a single-layered neural network while the second one has 3 hidden layers. They used information of 22283 genes extracted from the Gene Expression Omnibus repository. Their models achieved accuracies of $63.33 \%$ and $96.67 \%$ for single and multilayered DL architectures with a significant normalization test $(p>0.05)$. Jeyaraj and Samuel Nadar [142] used a regression-based DL algorithm to investigate hyperspectral images to diagnose oral cancer. Their system extracted patches for classification into normal, benign, and malignant classes using BioGPS, TCIA, and GDC datasets. For 100 malignant image patch training, they achieved an accuracy of $91.4 \%$ while for 500 malignant image patch training, they achieved an accuracy of $94.5 \%$. The authors in [143] proposed a DL method to study the relationship between genomic variations and traits. They analyzed 6083 sample exon sequencing files belonging to 12 cancer types. They used TCGA and 1000 Genomes Project. They performed both binary (cancer/healthy) and multiclass (12 classes) classifica- tion tasks using specific, total, and mixture models to achieve an accuracy of $97.47 \%, 70.08 \%$, and $94.7 \%$ for specific, mixture, and total specific models for the identification of cancer. Owais et al. [144] deployed a DL-based classification framework for the diagnosis of gastrointestinal diseases from endoscopic images. They deployed two datasets that are publicly available: Kvasir dataset and Gastrolab dataset. They followed a 2-step process. The classification network predicts the disease type in the first step, and then in the second step, the retrieval part shows the relevant cases. They performed multiclass (37 classes) classification using DenseNet transfer learning architecture, LSTM architecture, PCA, and KNN methods to achieve a correct recognition rate of $96.19 \%$ on this task. The authors in [145] proposed a CNN-based DL architecture for the multiclass (4 classes) classification of acute lymphoblastic leukemia. They used stained bone marrow images achieving an accuracy of $97.78 \%$. Kann et al. [146] deployed a 3D CNN model to identify nodal metastasis and tumor extranodal extension. Their dataset has $2875 \mathrm{CT}$ samples, 124 samples for validation and 131 samples for testing. They used a series of rotations and flipping technique to augment the datasets while achieving an AUC of 0.91. The authors in [147] proposed a DL approach to study the limited sample training problem from holographic images. They studied the classification of healthy and cancer cell lines. They used Generative Adversarial Networks (GANs) as the data augmentation method to train a large number of unclassified samples from sperm cells. Their model achieved an accuracy of $99 \%$ for healthy/primary cancer/metastatic cancer multiclass classification problems.

Table 5 displays a summary of the studies for the task of classification of cancers covered in this subsection.

4.5. Classification, Segmentation, Prediction, and Detection of Brain Tumors. Sun et al. [148] proposed a 3D fully 
TABLE 5: Summary of the studies for the classification of other types of cancer.

\begin{tabular}{|c|c|c|c|c|}
\hline Publication & Type(s) of cancer & Type of data & Methods & Performance \\
\hline$[125]$ & Colorectal cancer & Custom dataset & $\begin{array}{c}\text { VGG19, AlexNet, SqueezeNet } \\
\text { version 1.1, GoogLeNet, } \\
\text { ResNet-50 }\end{array}$ & $\begin{array}{c}\text { 98.7\% accuracy using VGG19 } \\
\text { model }\end{array}$ \\
\hline [126] & Colon cancer & Custom dataset & CNN model & $\begin{array}{r}\text { AUC }=0.998, \text { specificity }= \\
99.7 \% \text {, sensitivity }=100 \%\end{array}$ \\
\hline [127] & Gastric cancer & Custom datasets & DL models & $\begin{array}{l}\text { Accuracy of } 0.822 \text { in the } \\
\text { international validation cohort }\end{array}$ \\
\hline [128] & Bladder cancer & TCGA+custom dataset & DL models & Accuracy $($ custom $)=75 \%$ \\
\hline [129] & Lung cancer & TCGA+custom dataset & $\begin{array}{l}\text { Weakly supervised DL } \\
\text { algorithm }\end{array}$ & $\begin{array}{l}\text { Accuracy of } 97.3 \% \text { on the } \\
\text { custom dataset }\end{array}$ \\
\hline [130] & Prostate cancer & Custom dataset & DL methods & $\begin{array}{c}\text { Accuracy of } 92 \% \text { in cancerous/ } \\
\text { benign classification }\end{array}$ \\
\hline [131] & Skin cancer & Custom dataset & DL algorithms & $\begin{array}{l}\text { Positive predictive value of } \\
59.9 \%\end{array}$ \\
\hline [132] & Skin cancer & $\begin{array}{c}\text { HAM10000, Kaohsiung Chang Gung } \\
\text { Memorial Hospital }\end{array}$ & Lightweight DL algorithm & $\begin{array}{c}\text { Accuracy }=85.8 \% \\
\text { (HAM10000, multiclass } \\
\text { classification) }\end{array}$ \\
\hline [133] & Skin cancer & Custom dataset & Interpretable DL methods & $\begin{array}{c}\text { Accuracies between } 93.6 \% \text { and } \\
97.9 \%\end{array}$ \\
\hline [134] & Skin cancer & Custom dataset & CNN model & $\begin{array}{l}\text { Accuracy of } 82.95 \% \text { for } \\
\text { multiclass classification }\end{array}$ \\
\hline$[135]$ & Liver cancer & TCGA dataset & DL model & $\begin{array}{l}\text { High accuracy (abnormal/ } \\
\text { normal classification) }\end{array}$ \\
\hline$[136]$ & $\begin{array}{l}\text { Head and neck } \\
\text { cancer }\end{array}$ & Custom dataset & Inception version 3 & $\begin{array}{c}\text { Mean AUC was } 0.936 \text { based on } \\
\text { the testing set }\end{array}$ \\
\hline [137] & Pancreatic cancer & Three custom datasets & $\mathrm{CNN}$ architectures & Accuracy of 0.986 for test set 2 \\
\hline [138] & Multiple & Custom datasets & $\begin{array}{l}\text { ResNet-18, ResNet-34, } \\
\text { ResNet-50 }\end{array}$ & $\begin{array}{l}\text { Accuracy of } 94.90 \% \text { for } \\
\text { ResNet-50 architecture }\end{array}$ \\
\hline [139] & Breast cancer & $\begin{array}{l}\text { MITOS12, } 2016 \text { Tumor Proliferation } \\
\text { Assessment Challenge }\end{array}$ & $\mathrm{CNN}$ architecture & $F$-measure of 0.79 \\
\hline$[140]$ & Multiple & Custom dataset & $\begin{array}{l}\text { Multiple instance learning- } \\
\text { based DL system }\end{array}$ & Sensitivity $=100 \%$ \\
\hline [141] & $\begin{array}{l}\text { Blood and bone } \\
\text { marrow cancer }\end{array}$ & $\begin{array}{l}\text { Leukemia microarray gene data, Gene } \\
\text { Expression Omnibus repository }\end{array}$ & $\begin{array}{l}\text { Single-layer neural network, } \\
\text { 3-layered deep network }\end{array}$ & $96.67 \%$ for 3 layered model \\
\hline$[142]$ & Multiple & BioGPS data portal, TCIA, GDC dataset & $\begin{array}{l}\text { Regression-based partitioned } \\
\text { DL algorithm }\end{array}$ & Accuracy $=94.5 \%$ \\
\hline [143] & Multiple & TCGA, 1000 Genomes Project & DL algorithms & Accuracy $=97.47 \%$ \\
\hline$[144]$ & Multiple & Kvasir dataset, Gastrolab & $\begin{array}{c}\text { DL-based classification } \\
\text { network }\end{array}$ & Accuracy $=96.19 \%$ \\
\hline [145] & Multiple & Custom datasets & CNN model & Accuracy $=97.78 \%$ \\
\hline [146] & Multiple & Custom dataset & 3D CNN model & $\mathrm{AUC}=0.91$ \\
\hline [147] & Multiple & Custom dataset & GAN-based model & $90-99 \%$ accuracies \\
\hline
\end{tabular}

convolutional network-based multipathway architecture to extract features from MRI images from the BRATS 2019 challenge for the segmentation of brain tumor regions. They used the concept of dilated convolutions in each pathway to achieve a dice score of $0.89,0.78$, and 0.76 for whole tumor (WT), tumor core (TC), and enhancing tumor (ET) on the BRATS 2019 challenge, respectively. They used cropping, random slicing, and $z$-score normalization as the preprocessing methods. The authors in [149] proposed a novel architecture combining U-Net encoding and decoding sub- architecture, dilated convolutional feature extracting layers, and a residual module. Their proposed architecture achieved a dice score of $0.843,0.897$, and 0.906 and $0.798,0.902$, and 0.845 on ET, WT, and TC brain tumor subregions on BRATS 2018 and BRATS 2019 challenges, respectively. They used normalization and cropping techniques to preprocess the images. Khan et al. [150] utilized VGG16 and VGG19 transfer learning-based CNN models, partial least square covariance matrix, discrete cosine transform, and extreme learning machine to extract and classify features on BRATS 
2015, BRATS 2017, and BRATS 2018 challenge datasets to achieve an accuracy of $97.8 \%, 96.9 \%$, and $92.5 \%$ for BRATS 2015, BRATS 2017, and BRATS 2018 datasets, respectively. To preprocess the images before feeding them to the classification model, the authors used the histogram equalization approach. Pei et al. [151] proposed a joint deep and machine learning-based model for classification, segmentation, and prediction of brain tumors. Using a context-aware $\mathrm{CNN}$ architecture for segmentation, 3D CNN architecture for classification, and LASSO for prediction, the authors achieved a dice score of $0.821,0.895$, and 0.835 for ET, WT, and TC regions, respectively, on BRATS 2019 for the segmentation task, an accuracy of $58.6 \%$ for the survival prediction task on the BRATS 2019 dataset, and balanced accuracy of $63.9 \%$ on the test set for the 2019 Computational Precision Medicine Radiology-Pathology (2019 CPM-RadPath) challenge. The authors in [152] proposed a resourceefficient CNN model integrating memory connections and an adaptive dense block for the segmentation of brain tumors. They used the BRATS 2015 challenge dataset for the validation of their model and $z$-score normalization as a preprocessing method achieving a dice coefficient score of $0.858,0.816$, and 0.818 for WT, TC, and ET subregions. Badža and Barjaktarović [153] present a 22-layered CNN architecture for brain tumor classification of T1-weighted MRI images belonging to three categories: meningioma, glioma, and pituitary tumor. They normalize and resize the scans to $256 \times 256$ pixels followed by $90^{\circ}$ rotation and vertical flipping augmentation methods to synthetically increase the size of the dataset. The authors achieved an accuracy of 96.56\% for the multiclass classification task on a custom dataset. The authors in [154] proposed a transfer learningbased approach for segmentation and classification of brain tumors using Inception version 3-based features. They concatenated the CNN-based features with local binary pattern- (LBP-) based features. Contrast improvement is used as a preprocessing method. The authors achieved a dice score of $0.8373,0.937$, and 0.7994 for TC, WT, and ET subregions on the BRATS 2017 dataset and a dice score of $0.8834,0.912$, and 0.8184 for TC, WT, and ET subregions on the BRATS 2018 dataset. For the classification task, the authors achieved an average accuracy upward of $92 \%$ on BRATS 2013, BRATS 2014, BRATS 2017, and BRATS 2018 datasets. Rai et al. [155] proposed a U-Net-based DL model using skip connections for the classification, segmentation, and detection of tumors in brain MRI scans. They conducted their experiments on 120 patients of lowergrade glioma in TCGA database with 1373 scans for patients and 2556 scans for normal controls. The authors deployed cropping, resizing, global pixel normalization, horizontal flipping, flipping and rotation, random rotation, shift scale rotate, transposition, blurring, Gaussian blurring, random gamma, random brightness, and normalization as preprocessing and data augmentation methods. Their model achieved an accuracy of $99.7 \%$ on the classification task, a dice score of 0.9573 on the segmentation task, and a Jaccard index of 0.86 on the detection task. The authors in [156] compared and contrasted the performance of different transfer learning architectures for the binary classification of brain tumors into benign and malignant categories. They chose AlexNet, GoogLeNet, ResNet-50, ResNet-101, and SqueezeNet architectures for comparison. They employed a dataset of 224 benign category and 472 malignant category T1-weighted MRI images acquired from the TCIA public access repository. They used resizing, flipping, mirroring, salt noise addition, and rotation as preprocessing and data augmentation methods to achieve an accuracy of $99.04 \%$ using an AlexNet-type architecture. Feng et al. [157] developed a 3D U-Net model for brain tumor segmentation. They picked up an ensemble of models to extract features from brain MRI images on the BRATS 2018 challenge for segmentation and survival prediction. The authors achieved a dice score of $(0.7946,0.9114$, and 0.8304$)$ on (ET, WT, and TC) subregions for the segmentation task and an accuracy of $32.1 \%$ on the survival prediction task. The authors in [158] proposed an ensemble of deep CNN architectures integrating two and three paths of parallel models in a single model. They used 2D slices of brain MRI images from the BRATS 2013 dataset achieving a dice score of $(0.86,0.86$, and 0.88$)$ on (WT, TC, and ET) subregions. As a preprocessing step, they standardized the slices using the zero mean and unit variance normalization procedure. Naser and Deen [159] proposed a DL approach combining U-Net architecture, VGG16 transfer learning architecture, and a fully connected architecture for classification and segmentation of brain MRI images into lower-grade gliomas belonging to 110 patients. They used normalization, cropping, resizing, padding, rescaling, rotation, zooming, shifting, shearing, and flipping as preprocessing and data augmentation methods. Their approach achieved a dice score of 0.84 on the segmentation task and accuracy, sensitivity, and specificity of $92 \%$ on the binary classification (grade II/grade III) task. The authors in [160] proposed a multiscale 3D CNN architecture for the recognition and segmentation of 220 high- and 54 low-grade glioma MRI scans from the BRATS 2015 challenge dataset. As a preprocessing method, the authors used histogram matching to ensure consistency among gray levels. Their model achieved a dice score of 0.89 on the segmentation task, a sensitivity of 0.89 , and a specificity of 0.90 on the recognition task. Chang et al. [161] proposed a DL model combining average pooling and max pooling layers along with $1 \times 1$ kernels. They further combined this model with conditional random fields to optimize prediction results. The authors used the BRATS 2013 dataset to achieve a dice score of $(0.80,0.75$, and 0.71 ) on (WT, TC, and ET) subregions. As a preprocessing method, the authors used an intensity normalization method. The authors in [162] proposed a multiscale CNN model for the categorization of an MRI scan into healthy, meningioma, glioma, and pituitary tumor categories. The authors used 2D MRI images acquired from local hospitals in China to conduct their experiments. They achieved a dice score of $(0.894,0.779,0.813$, and 0.828$)$ on (meningioma, glioma, pituitary tumor, and average), respectively, and accuracy of $97.3 \%$ on the classification task. As preprocessing and data augmentation methods, the authors used pixel standardization and elastic transformation methods.

Table 6 displays a summary of the studies for the classification, segmentation, prediction, and detection of brain tumors covered in this subsection. 
TABLE 6: Summary of the studies for the classification, segmentation, prediction, and detection of brain tumors.

\begin{tabular}{|c|c|c|c|}
\hline Publication & Dataset(s) & Task(s) & Method \\
\hline [148] & BRATS 2019 & Segmentation & $\begin{array}{l}\text { 3D fully convolutional network-based } \\
\text { multipathway architecture }\end{array}$ \\
\hline [149] & $\begin{array}{l}\text { BRATS } 2018 \text { and } \\
\text { BRATS } 2019\end{array}$ & Segmentation & $\begin{array}{l}\text { Combination of U-Net encoding and } \\
\text { decoding subarchitecture, dilated } \\
\text { convolutional feature extracting layers, } \\
\text { and a residual module }\end{array}$ \\
\hline [150] & $\begin{array}{l}\text { BRATS 2015, } \\
\text { BRATS 2017, and } \\
\text { BRATS } 2018\end{array}$ & Classification & $\begin{array}{l}\text { VGG16 and VGG19 transfer learning- } \\
\text { based CNN models, partial least square } \\
\text { covariance matrix, discrete cosine } \\
\text { transform, and extreme learning machine }\end{array}$ \\
\hline [151] & $\begin{array}{l}\text { BRATS } 2019 \text { and } \\
2019 \text { CPM- } \\
\text { RadPath }\end{array}$ & $\begin{array}{l}\text { Classification, } \\
\text { segmentation, and } \\
\text { prediction }\end{array}$ & $\begin{array}{l}\text { Context-aware CNN architecture for } \\
\text { segmentation, 3D CNN architecture for } \\
\text { classification, and LASSO for prediction }\end{array}$ \\
\hline
\end{tabular}

BRATS 2013,

BRATS 2014, Segmentation and BRATS 2017, and classification BRATS 2018

Inception version $3+\mathrm{LBP}$

Resource-efficient CNN model with memory connections and an adaptive dense block

22-layered CNN architecture

Classification

Custom Classification

[155]
Custom

Classification, segmentation, and detection

Classification

TCIA public access repository

BRATS 2018

Segmentation and prediction

BRATS 2013 Segmentation

Custom

BRATS 2015

BRATS 2013

Segmentation and classification

Segmentation and classification

Segmentation

Segmentation and classification
U-Net-based DL model using skip connections

AlexNet, GoogLeNet, ResNet-50, ResNet-101, and SqueezeNet

3D U-Net model

Ensemble of deep CNN architectures

U-Net architecture, VGG16 transfer learning architecture, and a fully connected architecture

Multiscale 3D CNN architecture

DL model combining average pooling and max pooling layers along with $1 \times 1$ kernels

Multiscale Convolutional Neural Network

Performance

Dice score of $0.89,0.78$, and 0.76 for WT,

TC, and ET subregions, respectively

Dice score of $0.843,0.897$, and 0.906 and

$0.798,0.902$, and 0.845 on ET, WT, and

TC brain tumor subregions on BRATS

2018 and BRATS 2019 challenges, respectively

Accuracy of $97.8 \%, 96.9 \%$, and $92.5 \%$ for BRATS 2015, BRATS 2017, and BRATS 2018 datasets, respectively

Dice score of $0.821,0.895$, and 0.835 for

ET, WT, and TC regions, respectively, on BRATS 2019 for segmentation task,

accuracy of $58.6 \%$ for survival prediction task on BRATS 2019 dataset, and balanced accuracy of $63.9 \%$ on 2019 CPM-RadPath challenge

Dice coefficient score of $0.858,0.816$, and 0.818 for WT, TC, and ET subregions

\section{Accuracy of $96.56 \%$}

Dice score of $0.8373,0.937$, and 0.7994 for TC, WT, and ET subregions on

BRATS 2017; dice score of 0.8834, 0.912, and 0.8184 for TC, WT, and ET on

BRATS 2018; average accuracy upward of 92\% on BRATS 2013, BRATS 2014,

BRATS 2017, and BRATS 2018 datasets

Accuracy of $99.7 \%$ on the classification task, dice score of 0.9573 on the segmentation task, and Jaccard index of 0.86 on the detection task

An accuracy of $99.04 \%$ using AlexNettype architecture

Dice score of $0.7946,0.9114$, and 0.8304 on ET, WT, and TC, accuracy of $32.1 \%$

Dice score of $0.86,0.86$, and 0.88 on WT,

$$
\text { TC, and ET }
$$

Dice score of 0.84 ; accuracy, sensitivity, and specificity of $92 \%$ on the binary classification task

Dice score of 0.89 , sensitivity of 0.89 , and a specificity of 0.90

Dice score of $0.80,0.75$, and 0.71 on WT, $\mathrm{TC}$, and ET

Dice score of $0.894,0.779,0.813$, and 0.828 on meningioma, glioma, pituitary tumor, and average and an accuracy of $97.3 \%$

\section{Discussion}

The dynamics of cancer growth with respect to time are difficult to estimate. Precise measures can be made largely at the end of the cycle in cancer's evolution, when it is detached from the body. Ongoing mutations provide a rich history of clonal lineages which lead to changes in both genotype and phenotype. 
Psycho-oncology is a branch of oncology that deals directly with psychological and social issues. It deals with both emotional and psychobiological dimensions of cancer. However, there are still a number of obstacles in its wide adoption such as the dearth of medical practitioners as well as assessment tools and supporting instruments. It is important that both psychological and psychobiological factors influence the way cancers are treated. This domain must fulfill the demands for the availability of resources, support for caregivers and patients, and carving out new research directions for enthusiastic researchers [163, 164].

Research in AI has proven its worth in the support of medical decision-making. Due to the unknown nature of these algorithms, their widespread adoption is still limited. Explanatory AI algorithms provide a solution to this problem. However, performance issues might hinder their adoption as well. Robustness, local attribution, and completeness are three key properties of an explainable AI system. One way to get around this problem is to find strategies that optimally merge explainable and nonexplainable AI models. Some solutions that point in this direction are winning the confidence of clinicians by marking the regions in an image that are involved in AI predictions; another way is to attack or deceive the DL models through adversarial augmentations as it could potentially reveal the important features and discard the unimportant ones. There is a close link between interpretability and explainability. An explainable model is interpretable, but the reverse connection may not hold. A prediction relying on thousands of parameters is neither interpretable nor explainable $[165,166]$.

Precise DL model predictions are dependent on the availability of a large corpus of data (labelled or unlabelled), and it is a challenge to train it on a relatively small dataset. One way to look at this problem is through understanding the genetic evolution process. Gene transfer is the transfer of genetic information from a parent to its offspring. Genes encode genetic instructions (knowledge) from ancestors to descendants. The ancestors do not necessarily have better knowledge; yet, the evolution of knowledge across generations promotes a better learning curve for the descendants. There is a need for methods that can mimic this behaviour and use a limited number of examples to achieve their desired performance on different tasks [167]. Catastrophic forgetting is another problem limiting the performance of modern networks as they lack the ability to learn from continuous streams of data. The quality of the feature representation considerably determines the amount of forgetting. Boosting secondary information is the key to improving the transferability of features from old to new tasks without forgetting and is a promising direction for future work [168] especially for cancer diagnosis, prognosis, and prediction.

Despite the claims made by researchers, multiclass classification is an immensely difficult problem requiring a deeper understanding of human visual perception that moves beyond large datasets, and DL is perhaps necessary to solve many domain problems [169] including cancer diagnosis, prognosis, and prediction.

Another challenge that is worth mentioning is to find intricate hierarchical patterns from all forms of data such as labelled and unlabelled in a way that integrates information to perform visual inference. Unsupervised and semisupervised learning can help in this direction by offering potential solutions that help us in delving deeper into cancer pathogenesis and prediction tasks [170].

Can we use real-world images from another domain for calibration? Bridging the gap between cross-domain calibration and in-domain calibration is required to get optimal performance from neural networks. Techniques such as gram matrix similarity can be used as a criterion to select calibration datasets from a candidate pool to further improve performance [171]. This process can be used for effective feature construction in cancer diagnosis, prognosis, and prediction.

Modern DL object detection networks rely heavily on region proposal calculating algorithms to identify object locations. However, region proposal computation is a slow task. Faster region CNNs solve this problem by sharing convolutional layers with object detection subsystems. This process requires further research, and there is a need for improved computationally lightweight methods $[25,26]$. Cancer lesion detection can be improved by doing thorough research in this domain.

Modern DL networks rely heavily on global image statistics. This reliance can cause problems for these systems as shape and texture recognition is often better done at the local rather than the global level. Research in this domain can lead to better network generalization [172] holding the potential to improve cancer diagnosis, prognosis, and prediction.

Mitigating gradient explosion or decay in RNN training based on pondering over informative inputs to strengthen their contribution in the hidden state and finding computationally efficient ways for this purpose by suppressing noise in inputs or imposing novel constraints is a problem worth investigating [173].

Image recognition and image generation are two cornerstones of computer vision. While both are burgeoning fields, specialized techniques from both subareas can sometimes form a dichotomy. Historically, the field of DL was widely popularized in discriminative image classification with AlexNet architecture and image generation through GANs and Variational Autoencoders (VAEs). Novel data augmentation methods that force a network to pay attention to the moments extracted by layers of a deep network are a need of time [174] and can improve the performance of models in cancer diagnosis, prognosis, and prediction.

Further research should also target the discovery of novel objects (such as those having an aberrant organization, rare tumor, and foreign bodies), interpretable DL models (using influence functions or an attention mechanism), intraoperative decision-making, and tumor-infiltrating immune cell analysis. Some problems such as the appearance of whole-slide image as orderless texture-like image and color variation and artefacts are potentially hindering the performance of DL techniques [175] for cancer diagnosis, prognosis, and prediction.

Different types of imaging modalities like mammography, CT, MRI, and ultrasound have helped in the staging of cancer especially breast cancer. These systems have helped medical practitioners in the early identification of breast 
cancer [176]. For breast cancer, varying types of breast densities make masses very difficult to detect and classify in comparison to calcifications providing room for further research in this domain [177].

Other areas for potential research are scarcity of data, imbalanced datasets, missing data, and high dimensionality of patient data. Future work should be focused on testing and improving methods to achieve better performing DL models for cancer diagnosis, prognosis, and prediction tasks.

\section{Conclusion}

DL models have revolutionized the diagnosis and predictions of cancers. Data have been accepted in various forms and multiple sources. These models are excellent feature extractors, and their characteristics can improve cancer prognosis and prediction. Data augmentation is important for cancer diagnosis and prediction tasks to improve the final performance of systems. These methods will play a key role in making predictions about the cancer diagnosis and prediction tasks. However, further testing and validation are required on larger datasets for clinical applications. More research on data augmentation methods, learning in different domains such as frequency domain, and deploying novel architectures such as graph convolutional networks will likely improve their performance further.

\section{Data Availability}

No data were used to support this study.

\section{Conflicts of Interest}

No conflicts of interest exist between authors for the present study.

\section{Authors' Contributions}

A.B.T. conceived and designed the study. Y.K.M. and M.K.A.K. performed the analysis. All authors wrote and revised the draft manuscript.

\section{Acknowledgments}

We would like to acknowledge the group effort made in this research.

\section{References}

[1] P. S. Roy and B. J. Saikia, "Cancer and cure: a critical analysis," Indian Journal of Cancer, vol. 53, no. 3, pp. 441-442, 2016.

[2] L. A. Torre, R. L. Siegel, E. M. Ward, and A. Jemal, "Global cancer incidence and mortality rates and trends-an update," Cancer Epidemiology Biomarkers \& Prevention, vol. 25, no. 1, pp. 16-27, 2016.

[3] F. Abbas-Aghababazadeh, Q. Mo, and B. L. Fridley, "Statistical genomics in rare cancer," Seminars in Cancer Biology, vol. 61, pp. 1-10, 2020.
[4] J. M. Baust, Y. Rabin, T. J. Polascik et al., "Defeating cancers' adaptive defensive strategies using thermal therapies: examining cancer's therapeutic resistance, ablative, and computational modeling strategies as a means for improving therapeutic outcome," Technology in Cancer Research \& Treatment, vol. 17, 2018.

[5] R. Seelige, S. Searles, and J. D. Bui, "Innate sensing of cancer's non-immunologic hallmarks," Current Opinion in Immunology, vol. 50, pp. 1-8, 2018.

[6] A. V. Lichtenstein, "Genetic mosaicism and cancer: cause and effect," Cancer Research, vol. 78, no. 6, pp. 1375-1378, 2018.

[7] A. S. Balajee and M. P. Hande, "History and evolution of cytogenetic techniques: current and future applications in basic and clinical research," Mutation Research/Genetic Toxicology and Environmental Mutagenesis, vol. 836, Part A, pp. 3-12, 2018.

[8] S. Parida and D. Sharma, "The microbiome and cancer: creating friendly neighborhoods and removing the foes within," Cancer Research, vol. 81, no. 4, pp. 790-800, 2021.

[9] A. Dasgupta, M. Nomura, R. Shuck, and J. Yustein, "Cancer's Achilles' heel: Apoptosis and necroptosis to the rescue," International Journal of Molecular Sciences, vol. 18, no. 1, p. 23, 2017.

[10] V. Marx, "How to follow metabolic clues to find cancer's Achilles heel," Nature Methods, vol. 16, no. 3, pp. 221-224, 2019.

[11] T. Sepp, B. Ujvari, P. W. Ewald, F. Thomas, and M. Giraudeau, "Urban environment and cancer in wildlife: available evidence and future research avenues," Proceedings of the Royal Society B: Biological Sciences, vol. 286, no. 1894, article 20182434, 2019.

[12] C. Vrinten, L. M. McGregor, M. Heinrich et al., "What do people fear about cancer? A systematic review and metasynthesis of cancer fears in the general population," Psychooncology, vol. 26, no. 8, pp. 1070-1079, 2017.

[13] M. Karamanou, E. Tzavellas, K. Laios, M. Koutsilieris, and G. Androutsos, "Melancholy as a risk factor for cancer: a historical overview," JBUON, vol. 21, no. 3, pp. 756-759, 2016.

[14] Y. Lecun, Y. Bengio, and G. Hinton, "Deep learning," Nature, vol. 521, no. 7553, pp. 436-444, 2015.

[15] S. Ioffe and C. Szegedy, "Batch normalization: accelerating deep network training by reducing internal covariate shift," in Proceedings of the 32nd International Conference on Machine Learning, ICML 2015, pp. 448-456, Lille, France, 2015.

[16] G. Huang, Z. Liu, L. Van Der Maaten, and K. Q. Weinberger, "Densely connected convolutional networks," in Proceedings of the 30th IEEE Conference on Computer Vision and Pattern Recognition, CVPR, 2017, pp. 4700-4708, Honolulu, HI, USA, July 2017.

[17] M. T. Sadiq, H. Akbari, A. U. Rehman et al., "Exploiting feature selection and neural network techniques for identification of focal and nonfocal EEG signals in TQWT domain," Journal of Healthcare Engineering, vol. 2021, Article ID 6283900, 24 pages, 2021.

[18] M. Asif, W. U. Khan, H. M. R. Afzal et al., "Reduced-complexity LDPC decoding for next-generation IoT networks," Wireless Communications and Mobile Computing, vol. 2021, Article ID 2029560, 10 pages, 2021.

[19] A. R. Junejo, M. K. A. Kaabar, and S. Mohamed, "Future robust networks: current scenario and beyond for 6G," IMCC Journal of Science, vol. 11, no. 1, pp. 67-81, 2021. 
[20] K. Simonyan and A. Zisserman, "Very deep convolutional networks for large-scale image recognition," in Proceedings of the 3rd International Conference on Learning Representations, ICLR 2015-Conference Track Proceedings, pp. 1-14, San Diego, CA, USA, May 2015.

[21] C. Szegedy, W. Liu, Y. Jia et al., "Going deeper with convolutions," Proceedings of the IEEE Computer Society Conference on Computer Vision and Pattern Recognition, 2015, pp. 1-9, IEEE, Piscataway, NJ, USA, Boston, MA, USA, June 2015.

[22] F. Chollet, "Xception: deep learning with depthwise separable convolutions," in Proceedings of the 30th IEEE Conference on Computer Vision and Pattern Recognition, CVPR 2017, pp. 1251-1258, Honolulu, HI, USA, July 2017.

[23] K. He, X. Zhang, S. Ren, and J. Sun, "Deep residual learning for image recognition," in Proceedings of the IEEE Computer Society Conference on Computer Vision and Pattern Recognition, pp. 770-778, Las Vegas, NV, USA, June 2016.

[24] S. Hochreiter and J. Schmidhuber, "Long short-term memory," Neural Computation, vol. 9, no. 8, pp. 1735-1780, 1997.

[25] S. Ren, K. He, R. Girshick, and J. Sun, "Faster R-CNN: towards real-time object detection with region proposal networks," IEEE Transactions on Pattern Analysis and Machine Intelligence, vol. 39, no. 6, pp. 1137-1149, 2017.

[26] Z. Zhong, L. Sun, and Q. Huo, "An anchor-free region proposal network for Faster R-CNN-based text detection approaches," International Journal on Document Analysis and Recognition, vol. 22, no. 3, pp. 315-327, 2019.

[27] J. Donahue, Y. Jia, O. Vinyals et al., "DeCAF: a deep convolutional activation feature for generic visual recognition," in Proceedings of the 31st International Conference on Machine Learning, ICML 2014, pp. 647-655, Beijing, China, June 2014.

[28] B. Zoph, V. Vasudevan, J. Shlens, and Q. V. Le, "Learning transferable architectures for scalable image recognition," 2018, https://arxiv.org/abs/1707.07012v4.

[29] M. Tan and Q. V. Le, "EfficientNet: rethinking model scaling for convolutional neural networks," 2020, https://arxiv.org/ abs/1905.11946.

[30] P. Royston and D. G. Altman, "External validation of a Cox prognostic model: principles and methods," BMC medical research methodology, vol. 13, no. 1, p. 33, 2013.

[31] A. F. Connors, "A controlled trial to improve care for seriously III hospitalized patients," JAMA, vol. 274, no. 20, pp. 1591-1598, 1995.

[32] C. Curtis, S. P. Shah, S. F. Chin et al., "The genomic and transcriptomic architecture of 2,000 breast tumours reveals novel subgroups," Nature, vol. 486, no. 7403, pp. 346-352, 2012.

[33] M. Veta, Y. J. Heng, N. Stathonikos et al., "Predicting breast tumor proliferation from whole-slide images: the TUPAC16 challenge," Medical Image Analysis, vol. 54, pp. 111-121, 2019.

[34] I. C. Moreira, I. Amaral, I. Domingues, A. Cardoso, M. J. Cardoso, and J. S. Cardoso, "INbreast: toward a full-field digital mammographic database," Academic Radiology, vol. 19, no. 2, pp. 236-248, 2012.

[35] S. G. Armato, G. McLennan, L. Bidaut et al., "The Lung Image Database Consortium (LIDC) and Image Database Resource Initiative (IDRI): a completed reference database of lung nodules on CT scans," Medical Physics, vol. 38, no. 2, pp. 915931, 2011.

[36] A. A. Setio, A. Traverso, T. de Bel et al., "Validation, comparison, and combination of algorithms for automatic detection of pulmonary nodules in computed tomography images: the LUNA16 challenge," Medical Image Analysis, vol. 42, pp. 113, 2017.

[37] F. Spanhol, L. S. Oliveira, C. Petitjean, and L. Heutte, "A dataset for breast cancer histopathological image classification," IEEE Transactions on Biomedical Engineering, vol. 63, no. 7, pp. 1455-1462, 2016.

[38] T. Araújo, G. Aresta, E. Castro et al., "Classification of breast cancer histology images using convolutional neural networks," PLoS One, vol. 12, no. 6, article e0177544, 2017.

[39] G. Litjens, P. Bandi, B. Ehteshami Bejnordi et al., "1399 H\&Estained sentinel lymph node sections of breast cancer patients: the CAMELYON dataset," GigaScience, vol. 7, no. 6, 2018.

[40] B. Ehteshami Bejnordi, M. Veta, P. Johannes van Diest et al., "Diagnostic assessment of deep learning algorithms for detection of lymph node metastases in women with breast cancer," JAMA, vol. 318, no. 22, pp. 2199-2210, 2017.

[41] L. Roux, D. Racoceanu, N. Loménie et al., "Mitosis detection in breast cancer histological images an ICPR 2012 contest," Journal of Pathology Informatics, vol. 4, no. 1, p. 8, 2013.

[42] A. Risueno, C. Fontanillo, M. E. Dinger, and J. De Las Rivas, "GATExplorer: genomic and transcriptomic explorer; mapping expression probes to gene loci, transcripts, exons and ncRNAs," BMC Bioinformatics, vol. 11, no. 1, p. 221, 2010.

[43] T. Barrett, D. B. Troup, S. E. Wilhite et al., "NCBI GEO: archive for functional genomics data sets-10 years on," Nucleic Acids Research, vol. 39, pp. D1005-D1010, 2011.

[44] C. Wu, X. Jin, G. Tsueng, C. Afrasiabi, and A. I. Su, "BioGPS: building your own mash-up of gene annotations and expression profiles," Nucleic Acids Research, vol. 44, no. D1, pp. D313-D316, 2016.

[45] O. Diaz, K. Kushibar, R. Osuala et al., "Data preparation for artificial intelligence in medical imaging: a comprehensive guide to open-access platforms and tools," Physica Medica, vol. 83, pp. 25-37, 2021.

[46] P. Sudmant, T. Rausch, E. Gardner et al., "An integrated map of structural variation in 2,504 human genomes," Nature, vol. 526, no. 7571, pp. 75-81, 2015.

[47] K. Pogorelov, K. Randel, C. Griwodz et al., "Kvasir: a multiclass image-dataset for computer aided gastrointestinal disease detection," in ACM Multimedia Systems (MMSys'17), pp. 164-169, Taipei, Taiwan, 2017.

[48] E. Drelie Gelasca, J. Byun, B. Obara, and B. S. Manjunath, "Evaluation and benchmark for biological image segmentation," in 2008 15th IEEE International Conference on Image Processing, pp. 1816-1819, San Diego, CA, USA, 2008.

[49] B. H. Menze, A. Jakab, S. Bauer et al., "The Multimodal Brain Tumor Image Segmentation Benchmark (BRATS)," IEEE Transactions on Medical Imaging, vol. 34, no. 10, pp. 19932024, 2015.

[50] L. P. Petalidis, A. Oulas, M. Backlund et al., "Improved grading and survival prediction of human astrocytic brain tumors by artificial neural network analysis of gene expression microarray data," Molecular Cancer Therapeutics, vol. 7, no. 5, pp. 1013-1024, 2008.

[51] C. L. Chi, W. N. Street, and W. H. Wolberg, "Application of artificial neural network-based survival analysis on two breast cancer datasets," in AMIA Annual Symposium Proceedings 2007, pp. 130-134, Chicago, IL, USA, 2007. 
[52] Z. Huang, X. Zhan, S. Xiang et al., "SALMON: Survival Analysis Learning with Multi-Omics Neural Networks on breast cancer," Frontiers in Genetics, vol. 10, p. 166, 2019.

[53] H. Shimizu and K. I. Nakayama, "A 23 gene-based molecular prognostic score precisely predicts overall survival of breast cancer patients," EBioMedicine, vol. 46, pp. 150-159, 2019.

[54] R. Joshi and C. Reeves, "Beyond the Cox model: artificial neural networks for survival analysis part II," in Proceedings of the Eighteenth International Conference on Systems Engineering, pp. 179-184, Coventry, UK, May 2003.

[55] B. Jing, T. Zhang, Z. Wang et al., "A deep survival analysis method based on ranking," Artificial Intelligence in Medicine, vol. 98, pp. 1-9, 2019.

[56] J. Hao, Y. Kim, T.-K. Kim, and M. Kang, "PASNet: pathwayassociated sparse deep neural network for prognosis prediction from high-throughput data," BMC Bioinformatics, vol. 19, no. 1, p. 510, 2018.

[57] D. Sun, M. Wang, and A. Li, "A multimodal deep neural network for human breast cancer prognosis prediction by integrating multi-dimensional data," IEEE/ACM Transactions on Computational Biology and Bioinformatics, vol. 16, no. 3, pp. 841-850, 2019.

[58] K. Chaudhary, O. B. Poirion, L. Lu, and L. X. Garmire, "Deep learning-based multi-omics integration robustly predicts survival in liver cancer," Clinical Cancer Research, vol. 24, 2018.

[59] D. Bychkov, N. Linder, R. Turkki et al., "Deep learning based tissue analysis predicts outcome in colorectal cancer," Scientific Reports, vol. 8, no. 1, 2018.

[60] S. Wang, Z. Liu, Y. Rong et al., "Deep learning provides a new computed tomography-based prognostic biomarker for recurrence prediction in high-grade serous ovarian cancer," Radiotherapy and Oncology, vol. 132, pp. 171-177, 2019.

[61] T. Ching, X. Zhu, and L. X. Garmire, "Cox-nnet: an artificial neural network method for prognosis prediction of highthroughput omics data," PLoS Computational Biology, vol. 14, no. 4, 2018.

[62] J. L. Katzman, U. Shaham, A. Cloninger, J. Bates, T. Jiang, and Y. Kluger, "DeepSurv: personalized treatment recommender system using a Cox proportional hazards deep neural network," BMC Medical Research Methodology, vol. 18, no. 1, p. 24, 2018.

[63] P. Mobadersany, S. Yousefi, M. Amgad et al., "Predicting cancer outcomes from histology and genomics using convolutional networks," Proceedings of the National Academy of Sciences of the United Statetess of America, vol. 115, no. 13, pp. E2970-E2979, 2018.

[64] P. Courtiol, C. Maussion, M. Moarii et al., "Deep learningbased classification of mesothelioma improves prediction of patient outcome," Nature Medicine, vol. 25, no. 10, pp. 1519-1525, 2019.

[65] B. Liu, Y. Liu, X. Pan, M. Li, S. Yang, and S. C. Li, “DNA methylation markers for pan-cancer prediction by deep learning," Genes, vol. 10, no. 10, p. 778, 2019.

[66] M. H. Yap, G. Pons, J. Marti et al., “Automated breast ultrasound lesions detection using convolutional neural networks," IEEE Journal of Biomedical and Health Informatics, vol. 22, no. 4, pp. 1218-1226, 2018.

[67] M. H. Yap, M. Goyal, F. M. Osman et al., "Breast ultrasound lesions recognition: end-to-end deep learning approaches," Journal of Medical Imaging, vol. 6, pp. 1-7, 2019.
[68] Y. Liu, T. Kohlberger, M. Norouzi et al., "Artificial intelligence-based breast cancer nodal metastasis detection insights into the black box for pathologists," Archives of Pathology \& Laboratory Medicine, vol. 143, no. 7, pp. 859868, 2019.

[69] T. Mahmood, M. Arsalan, M. Owais, M. B. Lee, and K. R. Park, "Artificial intelligence-based mitosis detection in breast cancer histopathology images using Faster R-CNN and deep CNNs," Journal of Clinical Medicine, vol. 9, no. 3, p. 749, 2020.

[70] A. Anuranjeeta, K. K. Shukla, A. Tiwari, and S. Sharma, "Classification of histopathological images of breast cancerous and non cancerous cells based on morphological features," Biomedical and Pharmacology Journal, vol. 10, no. 1, pp. 353-366, 2017.

[71] D. Cai, X. Sun, N. Zhou, X. Han, and J. Yao, "Efficient mitosis detection in breast cancer histology images by RCNN," in Proceedings of the International Symposium on Biomedical Imaging, pp. 919-922, Venice, Italy, April 2019.

[72] J. Zhou, L. Y. Luo, Q. Dou et al., "Weakly supervised 3D deep learning for breast cancer classification and localization of the lesions in MR images," Journal of Magnetic Resonance Imaging, vol. 50, no. 4, pp. 1144-1151, 2019.

[73] M. A. Al-Antari, M. A. Al-Masni, M. T. Choi, S. M. Han, and T. S. Kim, "A fully integrated computer-aided diagnosis system for digital X-ray mammograms via deep learning detection, segmentation, and classification," International Journal of Medical Informatics, vol. 117, pp. 44-54, 2018.

[74] N. Nasrullah, J. Sang, M. S. Alam, M. Mateen, B. Cai, and $\mathrm{H}$. Hu, "Automated lung nodule detection and classification using deep learning combined with multiple strategies," Sensors, vol. 19, no. 17, p. 3722, 2019.

[75] O. Ozdemir, R. L. Russell, and A. A. Berlin, "A 3D probabilistic deep learning system for detection and diagnosis of lung cancer using low-dose CT scans," IEEE Transactions on Medical Imaging, vol. 39, no. 5, pp. 1419-1429, 2020.

[76] E. Shkolyar, X. Jia, T. C. Chang et al., "Augmented bladder tumor detection using deep learning," European Urology, vol. 76, no. 6, pp. 714-718, 2019.

[77] C. Fourcade, L. Ferrer, G. Santini et al., “Combining superpixels and deep learning approaches to segment active organs in metastatic breast cancer PET images," in 2020 42nd Annual International Conference of the IEEE Engineering in Medicine \& Biology Society (EMBC), pp. 1536-1539, Montreal, QC, Canada, 2020.

[78] M. Zhang, G. S. Young, H. Chen et al., "Deep-learning detection of cancer metastases to the brain on MRI," Journal of Magnetic Resonance Imaging, vol. 52, no. 4, pp. 1227-1236, 2020.

[79] J. Ma, S. Duan, Y. Zhang et al., "Efficient deep learning architecture for detection and recognition of thyroid nodules," Computational Intelligence and Neuroscience, vol. 2020, Article ID 1242781, 15 pages, 2020.

[80] A. Das, R. Acharya, S. S. Panda, and S. Sabut, "Deep learning based liver cancer detection using watershed transform and Gaussian mixture model techniques," Cognitive Systems Research, vol. 54, pp. 165-175, 2019.

[81] S. Lal, D. Das, K. Alabhya, A. Kanfade, A. Kumar, and J. Kini, "NucleiSegNet: robust deep learning architecture for the nuclei segmentation of liver cancer histopathology images," Computers in Biology and Medicine, vol. 128, article 104075, 2021. 
[82] P. Wang and A. C. S. Chung, "DoubleU-Net: colorectal cancer diagnosis and gland instance segmentation with textguided feature control," in Computer Vision - ECCV 2020 Workshops, pp. 338-354, Springer, 2020.

[83] J. Panic, A. Defeudis, S. Mazzetti et al., "A convolutional neural network based system for colorectal cancer segmentation on MRI images," in 2020 42nd Annual International Conference of the IEEE Engineering in Medicine \& Biology Society (EMBC), pp. 1675-1678, Montreal, QC, Canada, 2020.

[84] J. Juebin, H. Zhu, Z. Jindi et al., "Multiple U-Net-based automatic segmentations and radiomics feature stability on ultrasound images for patients with ovarian cancer," Frontiers in Oncology, vol. 10, p. 3428, 2021.

[85] T. Shibata, A. Teramoto, H. Yamada, N. Ohmiya, K. Saito, and H. Fujita, "Automated detection and segmentation of early gastric cancer from endoscopic images using mask R-CNN," Applied Sciences, vol. 10, no. 11, p. 3842, 2020.

[86] J. Wang and X. Liu, "Medical image recognition and segmentation of pathological slices of gastric cancer based on Deeplab v3+ neural network," Computer Methods and Programs in Biomedicine, vol. 207, article 106210, 2021.

[87] S. Shrestha, A. Alsadoon, P. W. C. Prasad, I. Seher, and O. H. Alsadoon, "A novel solution of using deep learning for prostate cancer segmentation: enhanced batch normalization," Multimedia Tools and Applications, vol. 80, no. 14, pp. 21293-21313, 2021.

[88] Z. Liu, C. Yang, J. Huang, S. Liu, Y. Zhuo, and X. Lu, “Deep learning framework based on integration of S-Mask R-CNN and Inception-v3 for ultrasound image-aided diagnosis of prostate cancer," Future Generation Computer Systems, vol. 114, pp. 358-367, 2021.

[89] T. Nemoto, N. Futakami, M. Yagi et al., "Simple low-cost approaches to semantic segmentation in radiation therapy planning for prostate cancer using deep learning with noncontrast planning CT images," Physica Medica, vol. 78, pp. 93-100, 2020.

[90] Y. Liang, D. Schott, Y. Zhang et al., "Auto-segmentation of pancreatic tumor in multi-parametric MRI using deep convolutional neural networks," Radiotherapy and Oncology, vol. 145, pp. 193-200, 2020.

[91] X. Chen, Z. Chen, J. Li, Y.-D. Zhang, X. Lin, and X. Qian, "Model-driven deep learning method for pancreatic cancer segmentation based on spiral-transformation," IEEE Transactions on Medical Imaging, p. 1, 2021.

[92] B. Huynh, K. Drukker, and M. Giger, "MO-DE-207B-06: computer-aided diagnosis of breast ultrasound images using transfer learning from deep convolutional neural networks," Medical Physics, vol. 43, pp. 3705-3705, 2016.

[93] M. Byra, M. Galperin, H. Ojeda-Fournier et al., "Breast mass classification in sonography with transfer learning using a deep convolutional neural network and color conversion," Medical Physics, vol. 46, no. 2, pp. 746-755, 2019.

[94] M. Byra, T. Sznajder, D. Korzinek et al., "Impact of ultrasound image reconstruction method on breast lesion classification with deep learning," 2018, https://arxiv.org/abs/1804 .02119 .

[95] A. Hijab, M. A. Rushdi, M. M. Gomaa, and A. Eldeib, "Breast cancer classification in ultrasound images using transfer learning," in Proceedings of the 2019 Fifth International Conference on Advances in Biomedical Engineering (ICABME), pp. 1-4, Tripoli, Lebanon, October 2019.
[96] O. Hadad, R. Bakalo, R. Ben-Ari, S. Hashoul, and G. Amit, "Classification of breast lesions using cross-modal deep learning," in 2017 IEEE 14th International Symposium on Biomedical Imaging (ISBI 2017), pp. 109-112, Melbourne, VIC, Australia, April 2017.

[97] D. S. Salem, R. M. Kamal, S. M. Mansour, L. A. Salah, and R. Wessam, "Breast imaging in the young: the role of magnetic resonance imaging in breast cancer screening, diagnosis and follow-up," Journal of Thoracic Disease, vol. 5, pp. 9-18, 2013.

[98] Q. Hu, H. M. Whitney, and M. L. Giger, "A deep learning methodology for improved breast cancer diagnosis using multiparametric MRI," Scientific Reports, vol. 10, 2020.

[99] M. Z. Alom, C. Yakopcic, M. S. Nasrin, T. M. Taha, and V. K. Asari, "Breast cancer classification from histopathological images with inception recurrent residual convolutional neural network," Journal of Digital Imaging, vol. 32, no. 4, pp. 605-617, 2019.

[100] N. Bayramoglu, J. Kannala, and J. Heikkila, “Deep learning for magnification independent breast cancer histopathology image classification," in Proceedings of the International Conference on Pattern Recognition, pp. 2440-2445, Cancun, Mexico, December 2016.

[101] D. M. Vo, N. Q. Nguyen, and S. W. Lee, "Classification of breast cancer histology images using incremental boosting convolution networks," Information Sciences, vol. 482, pp. 123-138, 2019.

[102] S. H. Kassani, P. H. Kassani, M. J. Wesolowski, K. A. Schneider, and R. Deters, "Classification of histopathological biopsy images using ensemble of deep learning networks," 2019, https://arxiv.org/abs/1909.11870.

[103] G. Murtaza, L. Shuib, G. Mujtaba, and G. Raza, “Breast cancer multi-classification through deep neural network and hierarchical classification approach," Multimedia Tools and Applications, vol. 79, no. 21-22, pp. 15481-15511, 2020.

[104] M. Toğaçar, K. B. Özkurt, B. Ergen, and Z. Cömert, "BreastNet: a novel convolutional neural network model through histopathological images for the diagnosis of breast cancer," Physica A: Statistical Mechanics and its Applications, vol. 545, article 123592, 2020.

[105] S. Alkassar, B. A. Jebur, M. A. M. Abdullah, J. H. Al-Khalidy, and J. A. Chambers, "Going deeper: magnification-invariant approach for breast cancer classification using histopathological images," IET Computer Vision, vol. 15, no. 2, pp. 151164, 2021.

[106] F. A. Spanhol, L. S. Oliveira, C. Petitjean, and L. Heutte, "Breast cancer histopathological image classification using convolutional neural networks," in Proceedings of the International Joint Conference on Neural Networks, pp. 25602567, Vancouver, BC, Canada, July 2016.

[107] Z. Han, B. Wei, Y. Zheng, Y. Yin, K. Li, and S. Li, "Breast cancer multi-classification from histopathological images with structured deep learning model," Scientific Reports, vol. 7, no. 1, 2017.

[108] D. Bardou, K. Zhang, and S. M. Ahmad, "Classification of breast cancer based on histology images using convolutional neural networks," IEEE Access, vol. 6, pp. 24680-24693, 2018.

[109] Z. Gandomkar, P. C. Brennan, and C. Mello-Thoms, "MuDeRN: multi-category classification of breast histopathological image using deep residual networks," Artificial Intelligence in Medicine, vol. 88, pp. 14-24, 2018. 
[110] Ü. Budak, Z. Cömert, Z. N. Rashid, A. Sengür, and M. Çıbuk, "Computer-aided diagnosis system combining FCN and BiLSTM model for efficient breast cancer detection from histopathological images," Applied Soft Computing, vol. 85, article 105765, 2019.

[111] K. George, S. Faziludeen, P. Sankaran, and J. K. Paul, “Deep learned nucleus features for breast cancer histopathological image analysis based on belief theoretical classifier fusion," in Proceedings of the IEEE Region 10 Annual International Conference, Proceedings/TENCON, pp. 344-349, Kochi, India, October 2019.

[112] P. J. Sudharshan, C. Petitjean, F. Spanhol, L. E. Oliveira, L. Heutte, and P. Honeine, "Multiple instance learning for histopathological breast cancer image classification," Expert Systems with Applications, vol. 117, pp. 103-111, 2019.

[113] A. Rakhlin, A. Shvets, V. Iglovikov, and A. A. Kalinin, “Deep convolutional neural networks for breast cancer histology image analysis," in Proceedings of the 15th International Conference, ICIAR 2018, pp. 737-744, Póvoa de Varzim, Portugal, June 2018.

[114] Y. Li, J. Wu, and Q. Wu, "Classification of breast cancer histology images using multi-size and discriminative patches based on deep learning," IEEE Access, vol. 7, pp. 2140021408, 2019.

[115] Shallu and R. Mehra, "Breast cancer histology images classification: training from scratch or transfer learning?," ICT Express, vol. 4, no. 4, pp. 247-254, 2018.

[116] K. Wadkar, P. Pathak, and N. Wagh, "Breast cancer detection using ANN network and performance analysis with SVM," International Journal of Computer Engineering and Technology, vol. 10, no. 3, pp. 75-86, 2019.

[117] V. Bevilacqua, A. Brunetti, A. Guerriero, G. F. Trotta, M. Telegrafo, and M. Moschetta, "A performance comparison between shallow and deeper neural networks supervised classification of tomosynthesis breast lesions images," Cognitive Systems Research, vol. 53, pp. 3-19, 2019.

[118] S. Sharma and R. Mehra, "Conventional machine learning and deep learning approach for multi-classification of breast cancer histopathology images-a comparative insight," Journal of Digital Imaging, vol. 33, no. 3, pp. 632-654, 2020.

[119] F. A. Spanhol, P. R. Cavalin, L. S. Oliveira, C. Petitjean, and L. Heutte, "Deep features for breast cancer histopathological image classification," in Proceedings of the 2017 IEEE International Conference on Systems, Man, and Cybernetics, SMC 2017, pp. 1868-1873, Banff, AB, Canada, October 2017.

[120] J. Li, J. Zhang, Q. Sun et al., "Breast cancer histopathological image classification based on deep second-order pooling network," in Proceedings of 2020 International Joint Conference on Neural Networks (IJCNN), pp. 1-7, Glasgow, UK, July 2020.

[121] S. U. Khan, N. Islam, Z. Jan, I. Ud Din, and J. J. P. C. Rodrigues, "A novel deep learning based framework for the detection and classification of breast cancer using transfer learning," Pattern Recognition Letters, vol. 125, pp. 1-6, 2019.

[122] X. Zhang, Y. Zhang, Q. Zhang et al., "Extracting comprehensive clinical information for breast cancer using deep learning methods," International Journal of Medical Informatics, vol. 132, article 103985, 2019.

[123] N. Naik, A. Madani, A. Esteva et al., “Deep learning-enabled breast cancer hormonal receptor status determination from base-level H\&E stains," Nature Communications, vol. 11, no. 1 , p. $5727,2020$.
[124] L. Shen, L. R. Margolies, J. H. Rothstein, E. Fluder, R. McBride, and W. Sieh, "Deep learning to improve breast cancer detection on screening mammography," Scientific Reports, vol. 9, no. 1, article 12495, 2019.

[125] J. N. Kather, J. Krisam, P. Charoentong et al., "Predicting survival from colorectal cancer histology slides using deep learning: a retrospective multicenter study," PLoS Medicine, vol. 16, no. 1, article e1002730, 2019.

[126] Y.Zeng, S. Xu, W. C. Chapman Jr. et al., "Real-time colorectal cancer diagnosis using PR-OCT with deep learning," Theranostics, vol. 10, no. 6, pp. 2587-2596, 2020.

[127] D. Dong, M. J. Fang, L. Tang et al., "Deep learning radiomic nomogram can predict the number of lymph node metastasis in locally advanced gastric cancer: an international multicenter study," Annals of Oncology, vol. 31, no. 7, pp. 912-920, 2020.

[128] A. C. Woerl, M. Eckstein, J. Geiger et al., "Deep learning predicts molecular subtype of muscle-invasive bladder cancer from conventional histopathological slides," European Urology, vol. 78, no. 2, pp. 256-264, 2020.

[129] X. Wang, H. Chen, C. Gan et al., "Weakly supervised deep learning for whole slide lung cancer image analysis," IEEE Transactions on Cybernetics, vol. 50, no. 9, pp. 3950-3962, 2020.

[130] D. Karimi, G. Nir, L. Fazli, P. C. Black, L. Goldenberg, and S. E. Salcudean, "Deep learning-based gleason grading of prostate cancer from histopathology images-role of multiscale decision aggregation and data augmentation," IEEE Journal of Biomedical and Health Informatics, vol. 24, no. 5, pp. 1413-1426, 2020.

[131] A. Dascalu and E. O. David, "Skin cancer detection by deep learning and sound analysis algorithms: a prospective clinical study of an elementary dermoscope," EBioMedicine, vol. 43, pp. 107-113, 2019.

[132] H. W. Huang, B. W. Hsu, C. H. Lee, and V. S. Tseng, "Development of a light-weight deep learning model for cloud applications and remote diagnosis of skin cancers," The Journal of Dermatology, vol. 48, no. 3, pp. 310-316, 2021.

[133] S. M. Thomas, J. G. Lefevre, G. Baxter, and N. A. Hamilton, "Interpretable deep learning systems for multi-class segmentation and classification of non-melanoma skin cancer," Medical Image Analysis, vol. 68, article 101915, 2021.

[134] A. Hekler, J. S. Utikal, A. H. Enk et al., "Superior skin cancer classification by the combination of human and artificial intelligence," European Journal of Cancer, vol. 120, pp. 114121, 2019.

[135] C. Sun, A. Xu, D. Liu, Z. Xiong, F. Zhao, and W. Ding, "Deep learning-based classification of liver cancer histopathology images using only global labels," IEEE journal of biomedical and health informatics, vol. 24, no. 6, pp. 1643-1651, 2020.

[136] S. Diao, J. Hou, H. Yu et al., "Computer-aided pathologic diagnosis of nasopharyngeal carcinoma based on deep learning," American Journal of Pathology, vol. 190, no. 8, pp. 16911700, 2020.

[137] K. L. Liu, T. Wu, P. T. Chen et al., “Deep learning to distinguish pancreatic cancer tissue from non-cancerous pancreatic tissue: a retrospective study with cross-racial external validation," The Lancet Digital Health, vol. 2, no. 6, pp. e303-e313, 2020.

[138] P. Korfiatis, T. L. Kline, D. H. Lachance, I. F. Parney, J. C. Buckner, and B. J. Erickson, "Residual deep convolutional 
neural network predicts mgmt methylation status," Journal of Digital Imaging, vol. 30, no. 5, pp. 622-628, 2017.

[139] N. Wahab, A. Khan, and Y. S. Lee, "Two-phase deep convolutional neural network for reducing class skewness in histopathological images based breast cancer detection," Computers in Biology and Medicine, vol. 85, pp. 86-97, 2017.

[140] G. Campanella, M. G. Hanna, L. Geneslaw et al., "Clinicalgrade computational pathology using weakly supervised deep learning on whole slide images," Nature Medicine, vol. 25, no. 8, pp. 1301-1309, 2019.

[141] E. Nazari, A. H. Farzin, M. Aghemiri, A. Avan, M. Tara, and H. Tabesh, "Deep learning for acute myeloid leukemia diagnosis," Journal of Medicine and Life, vol. 13, no. 3, pp. 382387, 2020.

[142] P. R. Jeyaraj and E. R. Samuel Nadar, "Computer-assisted medical image classification for early diagnosis of oral cancer employing deep learning algorithm," Journal of Cancer Research and Clinical Oncology, vol. 145, no. 4, pp. 829837, 2019.

[143] Y. Sun, S. Zhu, K. Ma et al., "Identification of 12 cancer types through genome deep learning," Scientific Reports, vol. 9, no. 1 , article 17256, 2019.

[144] M. Owais, M. Arsalan, T. Mahmood, J. K. Kang, and K. R. Park, "Automated diagnosis of various gastrointestinal lesions using a deep learning-based classification and retrieval framework with a large endoscopic database: model development and validation," Journal of Medical Internet Research, vol. 22, no. 11, article e18563, 2020.

[145] A. Rehman, N. Abbas, T. Saba, S. I. U. Rahman, Z. Mehmood, and H. Kolivand, "Classification of acute lymphoblastic leukemia using deep learning," Microscopy Research and Technique, vol. 81, no. 11, pp. 1310-1317, 2018.

[146] B. H. Kann, S. Aneja, G. V. Loganadane et al., "Pretreatment identification of head and neck cancer nodal metastasis and extranodal extension using deep learning neural networks," Scientific Reports, vol. 8, no. 1, article 14036, 2018.

[147] M. Rubin, O. Stein, N. A. Turko et al., "TOP-GAN: stain-free cancer cell classification using deep learning with a small training set," Medical Image Analysis, vol. 57, pp. 176-185, 2019.

[148] J. Sun, Y. Peng, Y. Guo, and D. Li, "Segmentation of the multimodal brain tumor image used the multi-pathway architecture method based on 3D FCN," Neurocomputing, vol. 423, pp. 34-45, 2021.

[149] J. Wang, J. Gao, J. Ren et al., “DFP-ResUNet:convolutional neural network with a dilated convolutional feature pyramid for multimodal brain tumor segmentation," Computer Methods and Programs in Biomedicine, vol. 208, p. 106208, 2021.

[150] M. A. Khan, I. Ashraf, M. Alhaisoni et al., "Multimodal brain tumor classification using deep learning and robust feature selection: a machine learning application for radiologists," Diagnostics, vol. 10, no. 8, p. 565, 2020.

[151] L. Pei, L. Vidyaratne, M. M. Rahman, and K. M. Iftekharuddin, "Context aware deep learning for brain tumor segmentation, subtype classification, and survival prediction using radiology images," Scientific Reports, vol. 10, no. 1, article 19726, 2020.

[152] Y. Ding, L. Gong, M. Zhang, C. Li, and Z. Qin, “A multi-path adaptive fusion network for multimodal brain tumor segmentation," Neurocomputing, vol. 412, pp. 19-30, 2020.
[153] M. M. Badža and M. Č. Barjaktarović, "Classification of brain tumors from MRI images using a convolutional neural network," Applied Sciences, vol. 10, no. 6, p. 1999, 2020.

[154] M. I. Sharif, J. P. Li, M. A. Khan, and M. A. Saleem, “Active deep neural network features selection for segmentation and recognition of brain tumors using MRI images," Pattern Recognition Letters, vol. 129, pp. 181-189, 2020.

[155] H. M. Rai, K. Chatterjee, and S. Dashkevich, "Automatic and accurate abnormality detection from brain MR images using a novel hybrid UnetResNext-50 deep CNN model," Biomedical Signal Processing and Control, vol. 66, article 102477, 2021.

[156] R. Mehrotra, R. A. Ansari, R. Agrawal, and R. S. Anand, "A transfer learning approach for AI-based classification of brain tumors," Machine Learning with Applications, vol. 2, article 100003, 2020.

[157] X. Feng, N. J. Tustison, S. H. Patel, and C. H. Meyer, "Brain tumor segmentation using an ensemble of 3D U-Nets and overall survival prediction using radiomic features," Frontiers in Computational Neuroscience, vol. 14, 2020.

[158] S. Sajid, S. Hussain, and A. Sarwar, "Brain tumor detection and segmentation in MR images using deep learning," Arabian Journal for Science and Engineering, vol. 44, no. 11, pp. 9249-9261, 2019.

[159] M. A. Naser and M. J. Deen, "Brain tumor segmentation and grading of lower-grade glioma using deep learning in MRI images," Computers in Biology and Medicine, vol. 121, p. 103758, 2020.

[160] W. Chen, B. Liu, S. Peng, J. Sun, and X. Qiao, "Computeraided grading of gliomas combining automatic segmentation and radiomics," International Journal of Biomedical Imaging, vol. 2018, Article ID 2512037, 11 pages, 2018.

[161] J. Chang, L. Zhang, N. Gu et al., "A mix-pooling CNN architecture with FCRF for brain tumor segmentation," Journal of Visual Communication and Image Representation, vol. 58, pp. 316-322, 2019.

[162] F. J. Díaz-Pernas, M. Martínez-Zarzuela, M. Antón-Rodríguez, and D. González-Ortega, "A deep learning approach for brain tumor classification and segmentation using a multiscale convolutional neural network," Healthcare, vol. 9, no. 2, p. 153, 2021.

[163] T. A. Graham and A. Sottoriva, "Measuring cancer evolution from the genome," The Journal of Pathology, vol. 241, no. 2, pp. 183-191, 2017.

[164] J. C. Holland, "Psycho-oncology: overview, obstacles and opportunities," Psycho-oncology, vol. 27, no. 5, pp. 13641376, 2018.

[165] G. Quellec, H. Al Hajj, M. Lamard, P. H. Conze, P. Massin, and B. Cochener, "ExplAIn: explanatory artificial intelligence for diabetic retinopathy diagnosis," Medical Image Analysis, vol. 72, article 102118, 2021.

[166] A. Rahnama and A. Tseng, "An adversarial approach for explaining the predictions of deep neural networks," in IEEE International Conference on Computer Vision and Pattern Recognition, pp. 3253-3262, Nashville, TN, USA, 2021.

[167] A. Taha, A. Shrivastava, and L. Davis, "Knowledge evolution in neural networks," in IEEE International Conference on Computer Vision and Pattern Recognition, pp. 1284312852, Nashville, TN, USA, 2021.

[168] S. Mittal, S. Galesso, and T. Brox, "Essentials for class incremental learning," in IEEE International Conference on 
Computer Vision and Pattern Recognition, pp. 3513-3522, Nashville, TN, USA, 2021.

[169] H. Ahmed, R. B. Wilbur, H. M. Bharadwaj, and J. M. Siskind, "Object classification from randomized EEG trials," in IEEE International Conference on Computer Vision and Pattern Recognition, pp. 3845-3854, Nashville, TN, USA, 2021.

[170] Y. LeCun, K. Kavukcuoglu, and C. Farabet, "Convolutional networks and applications in vision," in Proceedings of the ISCAS 2010-2010 IEEE International Symposium on Circuits and Systems: Nano-Bio Circuit Fabrics and Systems, pp. 253-256, Paris, France, May 2010.

[171] H. Yu, L. Yang, and H. Shi, "Is in-domain data really needed? A pilot study on cross-domain calibration for network quantization," in IEEE International Conference on Computer Vision and Pattern Recognition, pp. 3043-3052, Nashville, TN, USA, 2021.

[172] O. Nuriel, S. Benaim, and L. Wolf, "Permuted AdaIN: reducing the bias towards global statistics in image classification," in IEEE International Conference on Computer Vision and Pattern Recognition, pp. 9482-9491, Nashville, TN, USA, 2021.

[173] A. Kag and V. Saligrama, "Time adaptive recurrent neural network," in IEEE International Conference on Computer Vision and Pattern Recognition, pp. 15149-15158, Nashville, TN, USA, 2021.

[174] B. Li, F. Wu, S.-N. Lim et al., "On feature normalization and data augmentation," in IEEE International Conference on Computer Vision and Pattern Recognition, pp. 1238312392, Nashville, TN, USA, 2021.

[175] D. Komura and S. Ishikawa, "Machine learning methods for histopathological image analysis," Computational and Structural Biotechnology Journal, vol. 16, pp. 34-42, 2018.

[176] C. Kaushal, S. Bhat, D. Koundal, and A. Singla, "Recent trends in computer assisted diagnosis (cad) system for breast cancer diagnosis using histopathological images," IRBM, vol. 40, no. 4, pp. 211-227, 2019.

[177] S. J. S. Gardezi, A. Elazab, B. Lei, and T. Wang, "Breast cancer detection and diagnosis using mammographic data: systematic review," Journal of Medical Internet Research, vol. 21, no. 7, article e14464, 2019. 